DTP-92/17

April 1992

\title{
EULER HIERARCHIES AND UNIVERSAL EQUATIONS
}

\author{
D.B. FAIRLIE and J. GOVAERTS \\ Department of Mathematical Sciences \\ University of Durham, Durham DH1 3LE, England
}

\begin{abstract}
Finite Euler hierarchies of field theory Lagrangians leading to universal equations of motion for new types of string and membrane theories and for classical topological field theories are constructed. The analysis uses two main ingredients. On the one hand, there exists a generic finite Euler hierarchy for one field leading to a universal equation which generalises the Plebanski equation of self-dual four dimensional gravity. On the other hand, specific maps are introduced between field theories which provide a "triangular duality" between certain classes of arbitrary field theories, classical topological field theories and generalised string and membrane theories. The universal equations, which derive from an infinity of inequivalent Lagrangians, are generalisations of certain reductions of the Plebanski and KdV equations, and could possibly define new integrable systems, thus in particular integrable membrane theories. Some classes of solutions are constructed in the general case. The general solution to some of the universal equations is given in the simplest cases.
\end{abstract}




\section{Introduction}

In two recent papers ${ }^{[1,2]}$, the first with A. Morozov, hierarchies of Lagrangian field theories with the following properties were introduced.

i) In any of these hierarchies, the Lagrangian at any given level - except of course at the first level - is essentially proportional to the equations of motion of the Lagrangian at the previous level (hence the name Euler hierarchies).

ii) The proportionality factor mentioned in i) is essentially the very first Lagrangian in the hierarchy.

iii) In any of these hierarchies, Lagrangians depend on fields only through their first and second derivatives, but not on derivatives of higher order nor on the fields themselves. The first Lagrangian only depends on first derivatives of the fields. The dependence of each of the other Lagrangians on second derivatives is multilinear, and of order equal to the number of times an equation of motion has been taken to reach that level in the hierarchy.

iv) All these hierarchies are finite, i.e. the iterative procedure implied by i) - iii) terminates after a finite number of steps.

v) For each hierarchy, the last non trivial equations of motion are universal, namely, up to a factor, they are independent of the initial Lagrangian out of which the hierarchy is constructed. The associated infinite number of conserved charges - corresponding to the freedom in the choice of initial Lagrangian - suggests the possible integrability of these universal equations (equations of motion are indeed always current conservation equations for Lagrangians without an explicit dependence on fields).

Specifically, hierarchies with these properties were shown ${ }^{[1,2]}$ to exist in the following cases:

1) a single field $\phi$ in $d$ dimensions, with an arbitrary initial Lagrangian (function of first derivatives only) ${ }^{[1]}$,

2) a single field $\phi$ in $(d+1)$ dimensions, the initial Lagrangian now being an arbitrary homogeneous weight one function of its arguments ${ }^{[1]}$,

3) $(d+1)$ fields $\phi^{a}$ in $d$ dimensions, with an arbitrary reparametrisation invariant initial $\operatorname{Lagrangian}^{[2]}$.

The hierarchies associated with these three cases terminate after $d$ steps, with the following universal equations:

Case 1)

$$
\operatorname{det} \phi_{i j}=0 \text {, }
$$

Case 2)

$$
\operatorname{det}\left(\begin{array}{cc}
0 & \phi_{j} \\
\phi_{i} & \phi_{i j}
\end{array}\right)=0,
$$


Case 3)

$$
\operatorname{det}\left(J_{a} \phi_{i j}^{a}\right)=0 .
$$

Here, $\phi_{i}$ and $\phi_{i j}$ denote the partial derivatives $\left(\partial \phi / \partial x_{i}\right),\left(\partial^{2} \phi /\left(\partial x_{i} \partial x_{j}\right)\right)$ of the field $\phi$ with respect to the $d$ or $(d+1)$ coordinates $x_{i}$. (The same applies of course to the fields $\phi^{a}$, and obviously the indices $i$ and $j$ in the equations above refer to lines and columns respectively of the corresponding matrices. The usual summation convention over repeated indices is assumed throughout). In (1.3), the quantities $J_{a}$ are the Jacobians

$$
J_{a}=(-1)^{d} \epsilon_{a b_{1} b_{2} \cdots b_{d}} \phi_{1}^{b_{1}} \phi_{2}^{b_{2}} \ldots \phi_{d}^{b_{d}} .
$$

Eq.(1.1) is a generalisation to $d$ dimensions of a particular reduction (corresponding to $(1.1)$ for $(d=2))$ of the Plebanski equation ${ }^{[3]}$ for self-dual gravity in four dimensions, which is well known to be an integrable system. Eq.(1.2) is a generalisation of the original two dimensional Bateman equation ${ }^{[4,5]}$ (corresponding to $(1.2)$ with $(i=1,2)$ ) which is also known to be integrable ${ }^{[5,1]}$. Finally, $(1.3)$ is a generalisation to a $(d-1)$-dimensional membrane in a $(d+1)$-dimensional spacetime of the (universal) equation of motion for a parametrised particle in a flat two dimensional spacetime (corresponding to $(d=1)$ ), the latter clearly being also integrable. Note that (1.3) includes a universal equation for a string theory in three dimensions $(d=2)$, and a universal equation for a membrane theory in four dimensions $(d=3)$.

Remarkably, the three classes of universal equations above are invariant under arbitrary linear $G L(n)$ transformations in the variables $x_{i}$ as well as in the fields $\phi^{a}$, even though neither the initial nor the successive Lagrangians in the corresponding hierarchies would generally possess these symmetries. The equations above thus provide examples of equations of motion admitting an infinite number of Lagrangians, with symmetry properties that these Lagrangians need not possess!

Moreover, in cases 2) and 3), the properties required of the initial Lagrangians extend to the whole of the resulting hierarchies. Namely, in case 3), reparametrisation invariance in the coordinates $x_{i}$ for the field theory defined by the initial Lagrangian ensures ${ }^{[2]}$ the same invariance property for all field theories defined by the hierarchy. Thus in particular, the universal equation (1.3) is reparametrisation covariant in the variables $x_{i}$, as is desirable of any string or membrane theory. In case 2), weight one homogeneity of the initial Lagrangian ensures ${ }^{[1]}$ that all equations of motion of the hierarchy are invariant under arbitrary redefinitions of the field $\phi$. Thus in particular, the universal Bateman equation (1.2) possesses this property of general covariance in the field $\phi$. This notion of classical general covariance of equations of motion under arbitrary field redefinitions, i.e. general covariance on the space of solutions, may obviously be extended to the case of many fields $\phi^{a}$. Identifying these fields with coordinates in a target space, field theories leading to such generally covariant equations of motion provide a class of theories halfway between ordinary field theories and quantum topological field theories ${ }^{[6]}$. Namely, such theories correspond to classical topological field theories whose space of classical solutions falls into diffeomorphic topological classes of the possible target manifolds parametrised by the fields $\phi^{a}$. 
Actually, a generally covariant generalisation of the Bateman equation (1.2) to many fields was given in Ref.[1], when the number of fields $\phi^{a}$ is less than the number of coordinates $x_{i}$. Moreover, these equations were conjectured ${ }^{[1]}$ to be universal and to follow from a hierarchy construction of the type described in i) - v) above. In compact form, these generalised Bateman equations are given by

$$
\operatorname{det}\left(\begin{array}{cc}
0 & \phi_{j}^{a} \\
\phi_{i}^{b} & \phi_{i j}^{c} \lambda^{c}
\end{array}\right)=0
$$

Here, the determinant is that of a $(D+d) \times(D+d)$ matrix, with $D$ being the number of fields $\phi^{a}$ and $d$ the number of coordinates $x_{i}(D<d)$, and the indices $(a, i)$ and $(b, j)$ refer to lines and columns respectively (in particular, the entry " 0 " stands for the $D \times D$ null matrix). The quantities $\lambda^{c}$ are arbitrary coefficients, in terms of which the determinant is to be expanded. It is understood that (1.5) has to hold for all values of these coefficients. Hence, one obtains $\left(\begin{array}{l}d-1 \\ D-1\end{array}\right)$ equations - clearly generalising the Bateman equation (1.2) for one field - whose general covariance under arbitrary field redefinitions in $\phi^{a}$ is easily established. Even though these equations form an over determined set, except for $(D=1)$ or $(D=d-1)$ (the equations in the latter case were shown ${ }^{[1]}$ to be also universal), their space of solutions is non empty ${ }^{[1]}$. Functions $\phi^{a}\left(x_{i}\right)$ defined implicitly through the $D$ constraints

$$
x_{i} F_{i}^{a}\left(\phi^{b}\right)=c^{a},
$$

where $F_{i}^{a}\left(\phi^{b}\right)$ are arbitrary functions of the fields and $c^{a}$ arbitrary constants, indeed always provide solutions to (1.5). In fact, (1.6) defines ${ }^{[4,5]}$ the general solution to the Bateman equation in two dimensions.

In the present paper, the above results and conjecture are completed and extended in different directions. On the one hand, finite hierarchies with the characteristics i) - v) are shown to exist even when ii) is replaced by

iib) The proportionality factor mentioned in i) is essentially any function of the first derivatives of the fields.

Thus, the function by which the equations of motion at a given level are multiplied to define the Lagrangian at the next level may differ from level to level, and need not be the first Lagrangian in the hierarchy. As long as these multiplicative factors are functions of first derivatives of the fields only, as is the first Lagrangian, the hierarchy will be finite and terminate with universal equations of motion as described in v), namely, up to a factor, these equations are independent of the choice of multiplicative factors at every level. In particular in cases 1) - 3), the universal equations are still given by (1.1) - (1.3), provided the successive multiplicative factors in cases 2) and 3) possess the same properties as the first Lagrangian. In the following, the general construction leading to (1.1) will be referred to as the generic hierarchy, whereas the construction leading to (1.2) will be called the Bateman hierarchy.

On the other hand, some principles for the construction of classical topological field theories - as defined above - and of reparametrisation invariant field theories - without introducing a metric field - are presented. These constructions are generalisations of 
the map used in Ref.[2] to establish the existence of the hierarchy leading to (1.3) by relating it to the Bateman hierarchy. Namely, there exists a transformation, called here the $C$-map, taking any field theory of $p$ fields depending on $q$ variables into a classical topological field theory of $p$ fields depending on $(p+q)$ variables, with equations of motion generally covariant under arbitrary redefinitions of the $p$ fields. Similarly, there exists a transformation, called the $R$-map, taking any field theory of $p$ fields in $q$ variables into a reparametrisation invariant field theory of $(p+q)$ fields in $q$ variables. Moreover, these maps also define transformations between the equations of motion of the corresponding theories, thus providing relations between their respective solutions. Note that the C-map always produces a classical topological field theory with fewer fields than the number of coordinates, whereas the R-map always produces a reparametrisation invariant field theory with more fields than coordinates.

In particular, cases 1) - 3) are transformed into one another under applications of the C- and R-maps (these cases correspond to the situation described above with $(p=1)$ and $(q=d)$ arbitrary). Moreover, the C-map may be used to transform the generic hierarchy into a new hierarchy with (1.5) as its universal equations of motion. Under the R-map, one also obtains universal equations of motion for a $(q-1)$-dimensional membrane theory in a $(p+q)$-dimensional spacetime, thus in particular a universal string theory in $(p+2)$ dimensions. If the suggestion that these universal equations are integrable systems is correct, the R-map would thus lead to new types of integrable membrane (and string) theories of any dimension in a spacetime of arbitrary dimension, in contradistinction to membrane theories based on the Nambu-Goto action ${ }^{[7,8]}$ whose equations of motion are linear in second derivatives of the fields (these latter equations define an integrable system only in the string case $(q=2))$.

As will become clearer later on, classical topological field theories and reparametrisation invariant field theories - of the type constructed in this paper - may be viewed as being dual to each other. This duality is realised through the $\mathrm{C}$ - and $\mathrm{R}$-maps. In some sense, the universal reparametrisation invariant string and membrane equations of motion obtained here are generalisations of the usual Nambu-Goto equations, and their universal dual systems are classical topological field theories with fewer fields than coordinates generalising the original two dimensional Bateman equation ${ }^{[4]}$. In fact, this idea of duality, now explicitly realised through the $\mathrm{C}$ - and R-maps, was precisely one of the motivations in Ref.[1] which led to the discovery of finite Euler hierarchies with universal equations.

The paper is organised as follows. In sect.2, the relation between general covariance of equations of motion under field redefinitions, reparametrisation invariance and homogeneity properties of Lagrangians is discussed, extending some of the considerations of Refs.[1,2]. Sects.3 and 4 present the construction of C- and R-maps respectively, and discuss some of their basic properties. In sect.5, a new proof for the generic Euler hierarchy is presented, completing and generalising the main result of Ref.[1]. Using the generic hierarchy, sect. 6 shows how C- and R-maps can be applied to obtain finite Euler hierarchies of classical topological field theories and of reparametrisation invariant field theories, with the associated universal equations of motion. In particular, this includes the hierarchies leading to $(1.2),(1.3)$ and (1.5). In sect.7, some general classes of solutions to these universal equations are presented, taking advantage of the $\mathrm{C}$ - and $\mathrm{R}$-maps to relate solutions 
to different equations. Finally, conclusions are presented in sect.8. In an Appendix, definitions and properties of generalised determinants and traces of matrices, as they appear in the proof of the generic hierarchy, are collected for reference.

\section{Homogeneity, General Covariance and Reparametrisation Invariance}

In this section, we consider theories of $D$ fields $\phi^{a}$ depending on $d$ coordinates $x_{i}$, with Lagrangian densities $\mathcal{L}\left(\phi_{i}^{a}, \phi_{i j}^{a}\right)$ functions of first and second derivatives of the fields only. Even though the results of this section are valid for Lagrangians depending on derivatives of arbitrary higher order, the restriction to first and second derivatives only is sufficient for the purposes of this paper. The equation of motion for the field $\phi^{a}$ is thus given by

$$
\mathcal{E}_{a} \mathcal{L}\left[\phi^{a}\right]=0
$$

with the Euler operators $\mathcal{E}_{a}$ defined as

$$
\mathcal{E}_{a} \mathcal{L}\left[\phi^{a}\right]=\partial_{i}\left[\frac{\partial \mathcal{L}}{\partial \phi_{i}^{a}}\left(\phi_{i}^{a}, \phi_{i j}^{a}\right)\right]-\partial_{i} \partial_{j}\left[\frac{\partial \mathcal{L}}{\partial \phi_{i j}^{a}}\left(\phi_{i}^{a}, \phi_{i j}^{a}\right)\right]
$$

For most expressions in this paper, the arguments $\left[\phi^{a}\right]$ of an equation of motion will not be made explicit, unless they are different from the fields $\phi^{a}$.

First of all, consider Lagrangians with the following homogeneity property

$$
\mathcal{L}\left(R_{i}{ }^{j} \phi_{j}^{a}, R_{i}{ }^{k} R_{j}{ }^{l} \phi_{k l}^{a}+T_{i j}^{k} \phi_{k}^{a}\right)=\left(\operatorname{det} R_{i}{ }^{j}\right)^{\alpha} \mathcal{L}\left(\phi_{i}^{a}, \phi_{i j}^{a}\right)
$$

Here, $R_{i}{ }^{j}$ and $T_{i j}^{k}$ are arbitrary coefficients, and $\alpha$ is the weight of homogeneity of the Lagrangian. Differentiation of (2.3) with respect to the parameters $R_{i}{ }^{j}$ and $T_{i j}^{k}$ leads to the identities

$$
\begin{gathered}
\phi_{j}^{a} \frac{\partial \mathcal{L}}{\partial \phi_{i}^{a}}\left(\phi_{i}^{a}, \phi_{i j}^{a}\right)+\phi_{j k}^{a}\left[\frac{\partial \mathcal{L}}{\partial \phi_{i k}^{a}}\left(\phi_{i}^{a}, \phi_{i j}^{a}\right)+\frac{\partial \mathcal{L}}{\partial \phi_{k i}^{a}}\left(\phi_{i}^{a}, \phi_{i j}^{a}\right)\right]=\alpha \delta_{j}^{i} \mathcal{L}\left(\phi_{i}^{a}, \phi_{i j}^{a}\right), \\
\phi_{k}^{a} \frac{\partial \mathcal{L}}{\partial \phi_{i j}^{a}}\left(\phi_{i}^{a}, \phi_{i j}^{a}\right)=0 .
\end{gathered}
$$

Note that even though $\left(\phi_{i j}^{a}=\phi_{j i}^{a}\right)$, the dependence of the Lagrangian on $\phi_{i j}^{a}$ is not assumed to be necessarily identical to its dependence on $\phi_{j i}^{a}$, for fixed $(i \neq j)$.

Using these properties, a straightforward calculation shows that the equations of motion obey the following relations

$$
\phi_{i}^{a} \mathcal{E}_{a} \mathcal{L}\left[\phi^{a}\right]=(\alpha-1) \partial_{i} \mathcal{L}\left(\phi_{i}^{a}, \phi_{i j}^{a}\right)
$$

Therefore, when $(\alpha=1)$ there are $d$ identities among the $D$ equations of motion, leaving only $(D-d)$ independent equations of motion. In fact, the case $(\alpha=1)$ corresponds 
to a reparametrisation invariant action. Indeed, under a general reparametrisation of the coordinates of the form

$$
x_{i} \rightarrow y_{i}=y_{i}\left(x_{j}\right), \phi^{a}\left(x_{i}\right) \rightarrow \widetilde{\phi}^{a}\left(y_{i}\right)=\phi^{a}\left(x_{i}\right),
$$

we have

$$
\begin{aligned}
\frac{\partial \widetilde{\phi}^{a}}{\partial y_{i}} & =R_{i}{ }^{j} \frac{\partial \phi^{a}}{\partial x_{j}} \\
\frac{\partial^{2} \widetilde{\phi}^{a}}{\partial y_{i} \partial y_{j}} & =R_{i}{ }^{k} R_{j}{ }^{l} \frac{\partial^{2} \phi^{a}}{\partial x_{k} \partial x_{l}}+T_{i j}^{k} \frac{\partial \phi^{a}}{\partial x_{k}}
\end{aligned}
$$

with

$$
R_{i}{ }^{j}=\frac{\partial x_{j}}{\partial y_{i}}, \quad T_{i j}^{k}=\frac{\partial^{2} x_{k}}{\partial y_{i} \partial y_{j}} .
$$

Therefore, any Lagrangian obeying (2.3) scales under reparametrisations with a factor $\left(\operatorname{det} R_{i}{ }^{j}\right)^{\alpha}$. This factor cancels the Jacobian $\left(\operatorname{det} R_{i}{ }^{j}\right)^{-1}$ of the integration measure $\prod_{i} d x_{i}$ over the coordinates precisely for $(\alpha=1)$. Hence in this case, the identities

$$
\phi_{i}^{a} \mathcal{E}_{a} \mathcal{L}\left[\phi^{a}\right]=0
$$

are the Noether or Ward identities due to reparametrisation invariance, leaving only $(D-d)$ independent equations of motion. Incidentally, this conclusion also shows that a non trivial reparametrisation invariant field theory requires more fields than coordinates.

By analogy with (2.3), let us now consider Lagrangians with the following homogeneity property

$$
\mathcal{L}\left(\phi_{i}^{b} R_{b}{ }^{a}, \phi_{i j}^{b} R_{b}{ }^{a}+\phi_{i}^{b} T_{j}^{b a}+\phi_{j}^{b} T_{i}^{b a}\right)=\left(\operatorname{det} R_{a}{ }^{b}\right)^{\alpha} \mathcal{L}\left(\phi_{i}^{a}, \phi_{i j}^{a}\right) .
$$

Here, $R_{a}{ }^{b}$ and $T_{i}^{a b}$ are arbitrary coefficients, and $\alpha$ is the weight of homogeneity of the Lagrangian. Differentiation of (2.9) with respect to the coefficients $R_{a}{ }^{b}$ and $T_{i}^{a b}$ leads to the identities

$$
\begin{aligned}
\phi_{i}^{a} \frac{\partial \mathcal{L}}{\partial \phi_{i}^{b}}\left(\phi_{i}^{a}, \phi_{i j}^{a}\right)+\phi_{i j}^{a} \frac{\partial \mathcal{L}}{\partial \phi_{i j}^{b}}\left(\phi_{i}^{a}, \phi_{i j}^{a}\right) & =\alpha \delta_{b}^{a} \mathcal{L}\left(\phi_{i}^{a}, \phi_{i j}^{a}\right), \\
\phi_{j}^{a}\left[\frac{\partial \mathcal{L}}{\partial \phi_{i j}^{b}}\left(\phi_{i}^{a}, \phi_{i j}^{a}\right)+\frac{\partial \mathcal{L}}{\partial \phi_{j i}^{b}}\left(\phi_{i}^{a}, \phi_{i j}^{a}\right)\right] & =0 .
\end{aligned}
$$

On the other hand, differentiation of (2.9) with respect to $\phi_{i j}^{a}$ and $\phi_{i}^{a}$ leads to the further homogeneity properties:

$$
\begin{gathered}
\frac{\partial \mathcal{L}}{\partial \phi_{i j}^{a}}\left(\phi_{i}^{b} R_{b}{ }^{a}, \phi_{i j}^{b} R_{b}{ }^{a}+\phi_{i}^{b} T_{j}^{b a}+\phi_{j}^{b} T_{i}^{b a}\right)=\left(\operatorname{det} R_{a}^{b}\right)^{\alpha}\left(R^{-1}\right)_{a}{ }^{b} \frac{\partial \mathcal{L}}{\partial \phi_{i j}^{b}}\left(\phi_{i}^{a}, \phi_{i j}^{a}\right) \\
\frac{\partial \mathcal{L}}{\partial \phi_{i}^{a}}\left(\phi_{i}^{b} R_{b}{ }^{a}, \phi_{i j}^{b} R_{b}{ }^{a}+\phi_{i}^{b} T_{j}^{b a}+\phi_{j}^{b} T_{i}^{b a}\right)=\left(\operatorname{det} R_{a}{ }^{b}\right)^{\alpha}\left(R^{-1}\right)_{a}{ }^{b} \frac{\partial \mathcal{L}}{\partial \phi_{i}^{b}}\left(\phi_{i}^{a}, \phi_{i j}^{a}\right)- \\
-\left(\operatorname{det} R_{a}{ }^{b}\right)^{\alpha}\left(R^{-1}\right)_{a}{ }^{b} T_{j}^{b c}\left(R^{-1}\right)_{c}{ }^{d}\left[\frac{\partial \mathcal{L}}{\partial \phi_{i j}^{d}}\left(\phi_{i}^{a}, \phi_{i j}^{a}\right)+\frac{\partial \mathcal{L}}{\partial \phi_{j i}^{d}}\left(\phi_{i}^{a}, \phi_{i j}^{a}\right)\right] .
\end{gathered}
$$


Given these properties, consider now the transformation of the equations of motion (2.2) under arbitrary field redefinitions

$$
\varphi^{a}=F^{a}\left(\phi^{b}\right)
$$

We then have

$$
\varphi_{i}^{a}=\phi_{i}^{b} R_{b}{ }^{a}, \quad \varphi_{i j}^{a}=\phi_{i j}^{b} R_{b}^{a}+\phi_{i}^{b} T_{j}^{b a}+\phi_{j}^{b} T_{i}^{b a}
$$

where

$$
R_{a}{ }^{b}=\frac{\partial F^{b}}{\partial \phi^{a}}, \quad T_{i}^{b a}=\frac{1}{2} \phi_{i}^{c} \frac{\partial^{2} F^{a}}{\partial \phi^{b} \partial \phi^{c}} .
$$

Using the homogeneity properties (2.11) and the identities (2.10), a straightforward calculation then shows that the equations of motion $\mathcal{E}_{a} \mathcal{L}\left[\varphi^{a}\right]$ for the transformed fields $\varphi^{a}$ are given in terms of those for the fields $\phi^{a}$ by

$$
\mathcal{E}_{a} \mathcal{L}\left[\varphi^{a}\right]=\left(\operatorname{det} R_{a}{ }^{b}\right)^{\alpha}\left(R^{-1}\right)_{a}{ }^{b} \mathcal{E}_{b} \mathcal{L}\left[\phi^{a}\right]+\left(R^{-1}\right)_{a}{ }^{b} \frac{\partial\left(\operatorname{det} R_{a}{ }^{b}\right)^{\alpha}}{\partial \phi^{b}}(\alpha-1) \mathcal{L}\left(\phi_{i}^{a}, \phi_{i j}^{a}\right)
$$

Therefore, whenever the Lagrangian possesses the homogeneity property (2.9) with a weight $(\alpha=0)$ or $(\alpha=1)$, the equations of motion for the fields $\phi^{a}$ transform covariantly among themselves under arbitrary field redefinitions (2.12). This result is the complete generalisation of theorems in Ref.[1] establishing the same property in the case of a Lagrangian $\mathcal{L}\left(\phi_{i}^{a}\right)$ function only of first derivatives of the fields. In particular in the case of one field $(D=1)$, the equation of motion (2.2) is invariant when $(\alpha=1)$.

To conclude, we have thus shown that any Lagrangian $\mathcal{L}\left(\phi_{i}^{a}, \phi_{i j}^{a}\right)$ homogeneous in the sense of $(2.9)$ with a weight $(\alpha=1)$ or $(\alpha=0)$ defines a classical topological field theory. In fact, the case $(\alpha=1)$ plays a distinguished rôle, as will become clear in the next section when discussing the C-map.

\section{The C-Map}

To define the C-map, let us consider an arbitrary field theory Lagrangian $\mathcal{L}\left(y_{\alpha}^{a}, y_{\alpha \beta}^{a}\right)$ dependent on the first and second derivatives of $p$ fields $y^{a}\left(x_{\alpha}\right)$ functions of $q$ coordinates $x_{\alpha}$. The associated action is thus

$$
S\left[y^{a}\right]=\int \prod_{\alpha} d x_{\alpha} \mathcal{L}\left(\frac{\partial y^{a}}{\partial x_{\alpha}}, \frac{\partial^{2} y^{a}}{\partial x_{\alpha} \partial x_{\beta}}\right)
$$

whose variation under arbitrary field variations $\delta y^{a}$ is given, up to surface terms, by

$$
\delta S\left[y^{a}\right]=-\int \prod_{\alpha} d x_{\alpha} \delta y^{a} \mathcal{E}_{y^{a}} \mathcal{L}
$$


The Euler operators are

$$
\mathcal{E}_{y^{a}} \mathcal{L}=\partial_{\alpha} \frac{\partial \mathcal{L}}{\partial y_{\alpha}^{a}}-\partial_{\alpha} \partial_{\beta} \frac{\partial \mathcal{L}}{\partial y_{\alpha \beta}^{a}} .
$$

Let us now introduce $p$ additional coordinates $\phi^{a}$, and extend the coordinate dependence of the fields $y^{a}$ to also include a dependence on these new variables, i.e. $y^{a}\left(x_{\alpha}, \phi^{b}\right)$. Nevertheless, the corresponding field theory is still described by the original Lagrangian $\mathcal{L}\left(y_{\alpha}^{a}, y_{\alpha \beta}^{a}\right)$, with the action now given by

$$
S\left[y^{a}\right]=\int \prod_{\alpha} d x_{\alpha} \prod_{a} d \phi^{a} \mathcal{L}\left(\frac{\partial y^{a}}{\partial x_{\alpha}}, \frac{\partial^{2} y^{a}}{\partial x_{\alpha} \partial x_{\beta}}\right) .
$$

In particular, field variations still lead to

$$
\delta S\left[y^{a}\right]=-\int \prod_{\alpha} d x_{\alpha} \prod_{a} d \phi^{a} \delta y^{a} \mathcal{E}_{y^{a}} \mathcal{L},
$$

where the Euler operators $\mathcal{E}_{y^{a}}$ have the same definition as in (3.3), since the Lagrangian is independent of any derivatives of the fields $y^{a}$ with respect to the new variables $\phi^{a}$. In other words, these variables are irrelevant as far as the dynamical evolution of the fields $y^{a}$ is concerned; the equations of motion for both actions (3.1) and (3.4) are identical.

The intention is to invert the $\phi^{a}$ dependence of the fields $y^{a}$, and to consider $\phi^{a}$ as functions of $\left(x_{i}=\left(x_{\alpha}, y^{a}\right)\right)$, i.e. $\phi^{a}\left(x_{\alpha}, y^{a}\right)$. This requires that the matrix of derivatives $\left(\partial y^{a} / \partial \phi^{b}\right)$ be non singular, or equivalently that we have

$$
\operatorname{det} M_{a}{ }^{b} \neq 0,
$$

with $M_{a}{ }^{b}$ being the inverse matrix of derivatives $\left(\partial y^{a} / \partial \phi^{b}\right)$ keeping $x_{\alpha}$ fixed, namely

$$
M_{a}^{b}=\frac{\partial \phi^{b}}{\partial y^{a}} .
$$

The $\phi^{a}$ dependence of the fields $y^{a}$ may then be inverted, leading to configurations $\phi^{a}\left(x_{i}\right)$ for $(D=p)$ fields $\phi^{a}$ functions of $(d=p+q)$ variables $\left(x_{i}=\left(x_{\alpha}, y^{a}\right)\right)$. By direct substitution in the Lagrangian for the original field theory and its action (3.4), one then obtains a new Lagrangian field theory for the fields $\phi^{a}\left(x_{\alpha}, y^{b}\right)$. As is shown hereafter, the resulting theory is actually a classical topological field theory. A fortiori, this is to be expected. Indeed, as was pointed out above, the $\phi^{a}$ dependence in the original theory is irrelevant, so that the equations of motion for the fields $\phi^{a}$ in the new theory should be independent of the parametrisation used for these fields, namely their equations of motion should be generally covariant under arbitrary field transformations.

The series of operations described above define the C-map, which thus takes any field theory of $p$ fields in $q$ dimensions into a classical topological field theory of $(D=p)$ fields in $(d=p+q)$ dimensions. Note that the discussion is developed here for Lagrangians depending on first and second derivatives of fields only. However, it should be clear that 
the same considerations and conclusions apply in the case of Lagrangians depending on derivatives of arbitrary high order.

From the inversion $\phi^{a}\left(x_{\alpha}, y^{b}\right)$ defining the C-map, it is straightforward to derive the expressions that substitute for the derivatives of the original fields $y^{a}$. Namely, the derivatives $\left(\partial y^{a} / \partial x_{\alpha}\right)$ are substituted by the quantities

$$
Y_{\alpha}^{a}=-\frac{\partial \phi^{b}}{\partial x_{\alpha}}\left(M^{-1}\right)_{b}^{a}
$$

whereas the derivatives $\left(\partial^{2} y^{a} /\left(\partial x_{\alpha} \partial x_{\beta}\right)\right)$ are transformed into the quantities

$$
\begin{aligned}
Y_{\alpha \beta}^{a}=-\left[\frac{\partial^{2} \phi^{b}}{\partial x_{\alpha} \partial x_{\beta}}\right. & -\frac{\partial \phi^{c}}{\partial x_{\alpha}}\left(M^{-1}\right)_{c}{ }^{d} \frac{\partial^{2} \phi^{b}}{\partial y^{d} \partial x_{\beta}}-\frac{\partial \phi^{c}}{\partial x_{\beta}}\left(M^{-1}\right)_{c}{ }^{d} \frac{\partial^{2} \phi^{b}}{\partial y^{d} \partial x_{\alpha}}+ \\
& \left.+\frac{\partial \phi^{c}}{\partial x_{\alpha}}\left(M^{-1}\right)_{c} e^{e} \frac{\partial \phi^{d}}{\partial x_{\beta}}\left(M^{-1}\right)_{d}^{f} \frac{\partial^{2} \phi^{b}}{\partial y^{e} \partial y^{f}}\right]\left(M^{-1}\right)_{b}{ }^{a}
\end{aligned}
$$

Given these definitions, the Lagrangian for the transformed theory with fields $\phi^{a}\left(x_{i}\right)$ and coordinates $\left(x_{i}=\left(x_{\alpha}, y^{a}\right)\right)$ is simply obtained in terms of the original Lagrangian $\mathcal{L}\left(y_{\alpha}^{a}, y_{\alpha \beta}^{a}\right)$ as

$$
\mathcal{L}_{C}\left(\phi_{i}^{a}, \phi_{i j}^{a}\right)=\left(\operatorname{det} M_{a}^{b}\right) \mathcal{L}\left(Y_{\alpha}^{a}, Y_{\alpha \beta}^{a}\right) .
$$

With this identification, it is quite clear that the Lagrangian $\mathcal{L}_{C}\left(\phi_{i}^{a}, \phi_{i j}^{a}\right)$ possesses the homogeneity property (2.9) with a weight $(\alpha=1)$, thus establishing at once that the C-map has indeed produced a classical topological field theory.

By consideration of the variations of the actions for both field theories under variations of the fields $y^{a}$ and $\phi^{a}$, one also obtains the relation between the respective field equations of motion as

$$
\mathcal{E}_{\phi^{a}} \mathcal{L}_{C}=-\left(\operatorname{det} M_{a}^{b}\right)\left(M^{-1}\right)_{a}{ }^{b} \mathcal{E}_{y^{b}} \mathcal{L},
$$

where it is of course understood that the appropriate substitutions, such as those in (3.8) and (3.9), have to be applied on the right-hand side. Hence, solutions of either set of equations for which the non degeneracy condition (3.6) is satisfied are in one-to-one correspondence through the C-map. Note that the relations (3.11) also confirm the general covariance under field transformations of the equations for the fields $\phi^{a}$. It is the prefactor $\left(\left(\operatorname{det} M_{a}^{b}\right)\left(M^{-1}\right)_{a}{ }^{b}\right)$ in (3.11) which governs the transformation of these equations - and this prefactor indeed transforms covariantly under field redefinitions - since the equations $\left(\mathcal{E}_{y^{a}} \mathcal{L}\right)$ are independent of the parametrisation used for $\phi^{a}$.

Applying the C-map once more on the theory described by $\mathcal{L}_{C}\left(\phi_{i}^{a}, \phi_{i j}^{a}\right)$ does not produce a new classical topological field theory. Indeed, if the original field theory with Lagrangian $\mathcal{L}\left(y_{\alpha}^{a}, y_{\alpha \beta}^{a}\right)$ is itself a classical topological field theory in the sense of (2.9) with a weight $(\alpha=1)$, from that property and the identification (3.10) it is clear that the net effect of the C-map is to change the sign of the action by $(-1)^{p}$ and to substitute the fields $y^{a}$ by the fields $\phi^{a}$. In other words, no new field theory is produced, in agreement with the fact that the inversion defining the C-map is essentially a redefinition of the fields $y^{a}$, 
a transformation which does not affect the equations of motion for a classical topological field theory.

It is also possible to invert the C-map. Namely, given any classical topological field theory of $(D=p)$ fields dependent on $(d=p+q)$ variables, it is possible to define a new field theory of $p$ fields dependent on $q$ variables whose image under the C-map reproduces the initial classical topological field theory. Specifically, consider a Lagrangian $\mathcal{L}_{C}\left(\phi_{i}^{a}, \phi_{i j}^{a}\right)$ with $(D=p)$ fields $\phi^{a}\left(x_{i}\right)$ of $(d=p+q)$ coordinates $\left(x_{i}=\left(x_{\alpha}, y^{a}\right)\right)$ (there are thus $p$ coordinates $y^{a}$ and $q$ coordinates $x_{\alpha}$ ). This Lagrangian is assumed to obey the homogeneity property (2.9) with weight $(\alpha=1)$.

As before, in order to invert the $y^{a}$ dependence, consider field configurations such that

$$
\operatorname{det} N_{a}{ }^{b} \neq 0
$$

where the matrix $N_{a}^{b}$ is defined to be

$$
N_{a}^{b}=\frac{\partial y^{b}}{\partial \phi^{a}}
$$

The $y^{a}$ dependence of the fields $\phi^{a}$ may then be inverted, leading to the fields $y^{a}\left(x_{\alpha}, \phi^{b}\right)$. By direct substitution in the Lagrangian $\mathcal{L}_{C}$, one then obtains an action principle for these fields. However, due to the classical general covariance under field redefinitions of the system described by $\mathcal{L}_{C}$, one expects that any dependence on $\phi^{a}$ actually decouples in the equations of motion for the fields $y^{a}$. As is shown hereafter, such a decoupling indeed occurs. Hence, the inversion of the C-map as described above, takes any classical topological field theory of $p$ fields in $(p+q)$ dimensions into a field theory of $p$ fields in $q$ dimensions. Applying then the C-map on this latter field theory, one recovers the initial classical topological field theory described by $\mathcal{L}_{C}$. Here again, the same conclusions would apply to Lagrangians depending on field derivatives of higher order.

From the inversion $y^{a}\left(x_{\alpha}, \phi^{a}\right)$ defining the inverse C-map, it is straightforward to derive the expressions that substitute for the derivatives of the original fields $\phi^{a}$. The Lagrangian for the fields $y^{a}$ is then given by the function

$$
\left(\operatorname{det} N_{a}{ }^{b}\right) \mathcal{L}_{C}\left(\Phi_{i}^{b} R_{b}{ }^{a}, \Phi_{i j}^{b} R_{b}{ }^{a}+\Phi_{i}^{b} T_{j}^{b a}+\Phi_{j}^{b} T_{i}^{b a}\right),
$$

where the quantities $\Phi_{i}^{a}$ and $\Phi_{i j}^{a}$ are defined as

$$
\begin{gathered}
\Phi_{\alpha}^{c}=-\frac{\partial y^{c}}{\partial x_{\alpha}}, \quad \Phi_{a}^{c}=\delta_{a}^{c}, \\
\Phi_{\alpha \beta}^{c}=-\frac{\partial^{2} y^{c}}{\partial x_{\alpha} \partial x_{\beta}}, \quad \Phi_{\alpha b}^{c}=0, \quad \Phi_{a \beta}^{c}=0, \quad \Phi_{a b}^{c}=0,
\end{gathered}
$$

and the coefficients $R_{a}{ }^{b}$ and $T_{i}^{a b}$ by

$$
\begin{aligned}
R_{a}{ }^{b} & =\left(N^{-1}\right)_{a}{ }^{b} \\
T_{\alpha}^{b c} & =\left(N^{-1}\right)_{b}{ }^{d}\left[\frac{1}{2} \frac{\partial y^{e}}{\partial x_{\alpha}}\left(N^{-1}\right)_{e}{ }^{f} \frac{\partial^{2} y^{a}}{\partial \phi^{d} \partial \phi^{f}}-\frac{\partial^{2} y^{a}}{\partial x_{\alpha} \partial \phi^{d}}\right]\left(N^{-1}\right)_{a}{ }^{c}, \\
T_{a}^{b c} & =-\frac{1}{2}\left(N^{-1}\right)_{a}{ }^{e}\left(N^{-1}\right)_{b}{ }^{f} \frac{\partial^{2} y^{d}}{\partial \phi^{e} \partial \phi^{f}}\left(N^{-1}\right)_{d}{ }^{c} .
\end{aligned}
$$


However, owing to the homogeneity properties of the Lagrangian $\mathcal{L}_{C},(3.14)$ simplifies considerably, giving finally as Lagrangian for the fields $y^{a}$ the function

$$
\mathcal{L}\left(\frac{\partial y^{a}}{\partial x_{\alpha}}, \frac{\partial^{2} y^{a}}{\partial x_{\alpha} \partial x_{\beta}}\right)=\mathcal{L}_{C}\left(\Phi_{i}^{a}, \Phi_{i j}^{a}\right) .
$$

Since the quantities $\Phi_{i}^{a}$ and $\Phi_{i j}^{a}$ only involve derivatives of the fields $y^{a}$ with respect to the variables $x_{\alpha}$, the $\phi^{a}$ coordinates effectively decouple from the system, and one indeed obtains a theory of $p$ fields $y^{a}$ dependent on $q$ coordinates $x_{\alpha}$. Namely, suppressing from the action the integration over the variables $\phi^{a}$ does not modify the equations of motion for the fields $y^{a}$, as was already pointed out after (3.4) and (3.5) when considering the C-map.

Obviously, there is also a direct relation between the equations of motion for the two field theories related through the inverse C-map. This relation is essentially the inverse of (3.11), and reads

$$
\mathcal{E}_{y^{a}} \mathcal{L}=-\left(\operatorname{det} N_{a}^{b}\right)\left(N^{-1}\right)_{a}^{b} \mathcal{E}_{\phi^{b}} \mathcal{L}_{C}
$$

Finally, it is easy to verify that applying the C-map on the field theory described by $\mathcal{L}\left(y_{\alpha}^{a}, y_{\alpha \beta}^{a}\right)$ in (3.17) gives back the initial classical topological field theory described by $\mathcal{L}_{C}\left(\phi_{i}^{a}, \phi_{i j}^{a}\right)$. In particular, note that this conclusion also implies that any classical topological field theory of $p$ fields depending on $(p+q)$ coordinates and obeying the homogeneity property (2.9) with weight $(\alpha=1)$, may always be viewed as resulting from the C-map acting on some specific but otherwise arbitrary field theory Lagrangian of $p$ fields depending on $q$ coordinates. The C-map puts these two classes of fields theories in one-to-one correspondence.

\section{The R-Map}

To define the R-map, consider now an arbitrary field theory Lagrangian $\mathcal{L}\left(\frac{\partial \phi^{\mu}}{\partial z_{i}}, \frac{\partial^{2} \phi^{\mu}}{\partial z_{i} \partial z_{j}}\right)$ dependent on the first and second derivatives of $p$ fields $\phi^{\mu}\left(z_{i}\right)$ functions of $q$ coordinates $z_{i}$. The associated action is thus

$$
S\left[\phi^{\mu}\right]=\int \prod_{i} d z_{i} \mathcal{L}\left(\frac{\partial \phi^{\mu}}{\partial z_{i}}, \frac{\partial^{2} \phi^{\mu}}{\partial z_{i} \partial z_{j}}\right) .
$$

As always, variations of this action under field variations $\delta \phi^{\mu}$ are given, up to surface terms, by

$$
\delta S\left[\phi^{\mu}\right]=-\int \prod_{i} d z_{i} \delta \phi^{\mu} \mathcal{E}_{\phi^{\mu}} \mathcal{L}
$$

with the Euler operators

$$
\mathcal{E}_{\phi^{\mu}}=\frac{\partial}{\partial z_{i}}\left[\frac{\partial \mathcal{L}}{\partial\left(\frac{\partial \phi^{\mu}}{\partial z_{i}}\right)}\right]-\frac{\partial}{\partial z_{i}} \frac{\partial}{\partial z_{j}}\left[\frac{\partial \mathcal{L}}{\partial\left(\frac{\partial^{2} \phi^{\mu}}{\partial z_{i} \partial z_{j}}\right)}\right] .
$$


Let us now introduce $q$ arbitrary functions $x_{i}\left(z_{j}\right)$, and extend the field content of the theory to also include these extra degrees of freedom, while still keeping the same Lagrangian and action as in (4.1). Obviously, the equations of motion for $\phi^{\mu}$ remain as before, whereas those for the new fields $x_{i}$ are trivially satisfied since the Lagrangian is independent of these degrees of freedom.

Nevertheless, by inverting the $z_{i}$ dependence of the theory, one obtains a new field theory with reparametrisation invariance. For this purpose, consider field configurations such that

$$
\operatorname{det} M_{i}^{j} \neq 0
$$

where the matrix $M_{i}{ }^{j}$ is defined to be

$$
M_{i}^{j}=\frac{\partial z_{j}}{\partial x_{i}}
$$

The $z_{i}$ dependence of the fields $x_{i}$ may then be inverted, leading to a field theory of $(D=p+q)$ fields $\left(\phi^{a}=\left(\phi^{\mu}, z_{i}\right)\right)$ functions of $(d=q)$ variables $x_{i}$, with an action obtained from (4.2) by direct substitution. As shown hereafter, the resulting field theory is reparametrisation invariant in the coordinates $x_{i}$. A priori, this fact is to be expected since, on the one hand, the choice for the functions $x_{i}\left(z_{j}\right)$ is totally arbitrary, and on the other hand, their equations of motion are trivial, so that the transformed theory should indeed be independent of the choice of parametrisation in $x_{i}$. From a geometric point of view, the functions $\phi^{\mu}\left(z_{i}\right)$ define a $q$-dimensional subspace of the $(p+q)$-dimensional space spanned by the coordinates $\left(\phi^{a}=\left(\phi^{\mu}, z_{i}\right)\right)$. Introducing the fields $x_{i}\left(z_{j}\right)$ amounts to introducing an arbitrary intrinsic parametrisation of this subspace, without affecting its topological and geometrical properties as an embedded space. In other words, we are simply dealing with parametrised $(q-1)$-dimensional membrane theories in $(p+q)$ dimensions.

The series of operations described above define the R-map, which thus takes any field theory of $p$ fields in $q$ dimensions into a reparametrisation invariant field theory of $(D=p+q)$ fields in $(d=q)$ dimensions. Again, even though the discussion is developed for Lagrangians depending on first and second derivatives of the fields only, the same conclusions apply in the case of Lagrangians depending on derivatives of arbitrary high order.

From the inversion $\phi^{a}\left(x_{i}\right)$ defining the R-map, it is easy to derive the expressions that substitute for the derivatives of the original fields $\phi^{\mu}$ with respect to the variables $z_{i}$. Namely, the derivatives $\left(\partial \phi^{\mu} / \partial z_{i}\right)$ are replaced by

$$
Y_{i}^{\mu}=\left(M^{-1}\right)_{i}{ }^{j} \frac{\partial \phi^{\mu}}{\partial x_{j}}
$$

whereas the derivatives $\left(\partial^{2} \phi^{\mu} /\left(\partial z_{i} \partial z_{j}\right)\right)$ are substituted by the quantities

$$
Y_{i j}^{\mu}=\left(M^{-1}\right)_{i}{ }^{k}\left(M^{-1}\right)_{j}{ }^{l}\left[\frac{\partial^{2} \phi^{\mu}}{\partial x_{k} \partial x_{l}}-\frac{\partial z_{m}}{\partial x_{k} \partial x_{l}}\left(M^{-1}\right)_{m}{ }^{n} \frac{\partial \phi^{\mu}}{\partial x_{n}}\right] .
$$


Given these definitions, the Lagrangian for the fields $\left(\phi^{a}\left(x_{j}\right)=\left(\phi^{\mu}\left(x_{j}\right), z_{i}\left(x_{j}\right)\right)\right)$ and the coordinates $x_{i}$ is simply obtained in terms of the original Lagrangian $\mathcal{L}$ in (4.1) as

$$
\mathcal{L}_{R}\left(\frac{\partial \phi^{a}}{\partial x_{i}}, \frac{\partial^{2} \phi^{a}}{\partial x_{i} \partial x_{j}}\right)=\left(\operatorname{det} M_{i}^{j}\right) \mathcal{L}\left(Y_{i}^{\mu}, Y_{i j}^{\mu}\right)
$$

With this identification, it is straighforward to verify that the Lagrangian $\mathcal{L}_{R}$ obeys the homogeneity property (2.3) with a weight $(\alpha=1)$, thereby establishing at once that the R-map has indeed produced a reparametrisation invariant field theory.

The equations of motion of both theories are also in correspondence. However, we know from (2.8) that for the reparametrisation invariant theory there are only $p$ independent equations, the remaining $q$ equations being obtained from the Ward identities. Clearly, the independent equations are those for the $p$ fields $\phi^{\mu}$, which for both theories are related as

$$
\mathcal{E}_{\phi^{\mu}} \mathcal{L}_{R}=\left(\operatorname{det} M_{i}{ }^{j}\right) \mathcal{E}_{\phi^{\mu}} \mathcal{L}
$$

with of course the appropriate substitutions being implemented on the right-hand side. The remaining equations of motion for the $q$ fields $z_{i}\left(x_{j}\right)$ are simply obtained from the Ward identities, giving

$$
\begin{aligned}
\mathcal{E}_{z_{i}} \mathcal{L}_{R} & =-\left(M^{-1}\right)_{i}{ }^{j} \frac{\partial \phi^{\mu}}{\partial x_{j}} \mathcal{E}_{\phi^{\mu}} \mathcal{L}_{R} \\
& =-\left(\operatorname{det} M_{i}{ }^{j}\right)\left(M^{-1}\right)_{i}{ }^{j} \frac{\partial \phi^{\mu}}{\partial x_{j}} \mathcal{E}_{\phi^{\mu}} \mathcal{L} .
\end{aligned}
$$

These expressions also make it clear that under reparametrisations of the coordinates $x_{i}$, the equations of motion for the theory obtained through the R-map transform among themselves, in agreement with the reparametrisation invariance of that theory.

Applying the R-map once more on the theory described by $\mathcal{L}_{R}$ does not produce a new reparametrisation invariant theory. Indeed, if the original theory is already reparametrisation invariant, namely satisfies $(2.3)$ with weight $(\alpha=1)$, it is clear from $(4.6)-(4.8)$ that the net effect of the R-map is to substitute the $z_{i}$ dependence of the fields $\phi^{\mu}$ by a $x_{i}$ dependence, while the fields $z_{i}\left(x_{j}\right)$ that would otherwise arise from the R-map actually do not appear in the transformed Lagrangian. Of course, this is due to reparametrisation invariance, since the inversion defining the R-map is simply a reparametrisation in the coordinates $z_{i}$. The interest of the $\mathrm{R}$-map is that it produces in the general case a reparametrisation invariant field theory from one which is not.

Conversely, it is also possible to invert the R-map. Namely, given any reparametrisation invariant theory of $(D=p+q)$ fields $\left(\phi^{a}=\left(\phi^{\mu}, z_{i}\right)\right)$ dependent on $(d=q)$ coordinates $x_{i}$ with Lagrangian $\mathcal{L}_{R}\left(\frac{\partial \phi^{a}}{\partial x_{i}}, \frac{\partial^{2} \phi^{a}}{\partial x_{i} \partial x_{j}}\right)$ (there are thus $p$ fields $\phi^{\mu}$ and $q$ fields $z_{i}$, and the Lagrangian is assumed to obey $(2.3)$ with $(\alpha=1)$ ), there is a map producing a theory of $p$ fields in $q$ dimensions whose image under the R-map is the initial theory. Clearly, the inverse R-map is obtained by inverting the $x_{i}$ dependence of the fields $z_{i}$. Let us thus consider field configurations such that

$$
\operatorname{det} N_{i}^{j} \neq 0
$$


with the matrix $N_{i}{ }^{j}$ being

$$
N_{i}{ }^{j}=\frac{\partial x_{j}}{\partial z_{i}} .
$$

The $x_{i}$ dependence is then invertible, leading to the fields $\phi^{\mu}\left(z_{j}\right)$ and $x_{i}\left(z_{j}\right)$ with an action obtained from $\mathcal{L}_{R}$ by direct substitution. Clearly, due to reparametrisation invariance of the latter Lagrangian, one expects that the fields $x_{i}$ decouple from the system, leaving a theory of $p$ fields in $q$ dimensions. Acting with the R-map on the latter field theory reproduces again the initial reparametrisation invariant one.

Specifically, the Lagrangian obtained from the inverse R-map is simply

$$
\left(\operatorname{det} N_{i}{ }^{j}\right) \mathcal{L}_{R}\left(R_{i}{ }^{j} \Psi_{j}^{a}, R_{i}{ }^{k} R_{j}{ }^{l} \Psi_{k l}^{a}+T_{i j}^{k} \Psi_{k}^{a}\right),
$$

where we defined

$$
\begin{gathered}
\Psi_{i}^{\nu}=\frac{\partial \phi^{\nu}}{\partial z_{i}}, \quad \Psi_{i}^{j}=\delta_{i}^{j}, \\
\Psi_{i j}^{\rho}=\frac{\partial^{2} \phi^{\rho}}{\partial z_{i} \partial z_{j}}, \quad \Psi_{i j}^{k}=0,
\end{gathered}
$$

and

$$
\begin{aligned}
R_{i}{ }^{j} & =\left(N^{-1}\right)_{i}{ }^{j}, \\
T_{i j}^{k} & =-\left(N^{-1}\right)_{i}{ }^{l}\left(N^{-1}\right)_{j}{ }^{m} \frac{\partial^{2} x_{n}}{\partial z_{l} \partial z_{m}}\left(N^{-1}\right)_{n}{ }^{k} .
\end{aligned}
$$

However, owing to the homogeneity properties of the Lagrangian $\mathcal{L}_{R}$, (4.13) simplifies leaving for the transformed Lagrangian

$$
\mathcal{L}\left(\frac{\partial \phi^{\mu}}{\partial z_{i}}, \frac{\partial^{2} \phi^{\mu}}{\partial z_{i} \partial z_{j}}\right)=\mathcal{L}_{R}\left(\Psi_{i}^{a}, \Psi_{i j}^{a}\right) .
$$

Clearly, since the quantities $\Psi_{i}^{a}$ and $\Psi_{i j}^{a}$ involve derivatives only of the fields $\phi^{\mu}\left(z_{j}\right)$, the fields $z_{i}\left(x_{j}\right)$ have indeed decoupled, leaving a theory of $p$ fields in $q$ dimensions with the Lagrangian (4.16). Obviously, the equations of motion for both theories are also in correspondence, the relation being

$$
\mathcal{E}_{\phi^{\mu}} \mathcal{L}=\left(\operatorname{det} N_{i}{ }^{j}\right) \mathcal{E}_{\phi^{\mu}} \mathcal{L}_{R}
$$

Finally, it is straightforward to verify that the R-map acting on the theory defined by (4.16) gives back the original field theory described by $\mathcal{L}_{R}$. In particular, this result implies that any reparametrisation invariant theory of $(p+q)$ fields in $q$ dimensions, with a Lagrangian obeying the homogeneity property (2.3) with $(\alpha=1)$, may always be viewed as resulting from the R-map acting on some specific but otherwise arbitrary theory of $p$ fields in $q$ dimensions. The R-map puts these two classes of theories in one-to-one correspondence.

Having introduced the $\mathrm{C}$ - and R-maps and the associated inverse transformations, one may wonder what happens when these maps are applied in succession. We have already seen that applying twice the C-map or the R-map only reproduces the result of the $\mathrm{C}$ - 
or the R-map applied once. This only leaves the successive application of the two maps alternatively. For convenience, given a Lagrangian $\mathcal{L}$ (with its field content implicit), let us symbolically denote the results of the $\mathrm{C}$ - and $\mathrm{R}$-maps as

$$
\mathcal{L}_{C}=\mathcal{O}_{C} \mathcal{L}, \quad \mathcal{L}_{R}=\mathcal{O}_{R} \mathcal{L}
$$

Thus, one may still act on $\mathcal{L}_{C}$ with the R-map and on $\mathcal{L}_{R}$ with the C-map. However, due to the respective properties - under field redefinitions or reparametrisations - of these Lagrangians, it is possible to show that the net result of these transformations is to lead essentially to the following identifications:

$$
\mathcal{O}_{R} \mathcal{L}_{C}=\mathcal{L}_{R}, \quad \mathcal{O}_{C} \mathcal{L}_{R}=\mathcal{L}_{C}
$$

Namely, in either case the resulting system possesses one of the two properties for all of the associated variables, and the other property for some subset only of the other set of associated variables. Taking advantage of both properties under field redefinitions and reparametrisations, it becomes possible to set equal those fields and those coordinates for which both properties are operative, so that these field degrees of freedom effectively decouple from the system. This decoupling then only leaves the field theories whose content is expressed through (4.19). Of course, the counting of the number of equations of motion and Ward identities in all cases agrees with this conclusion, as may easily be checked.

We have therefore obtained the following situation. Given an arbitrary Lagrangian $\mathcal{L}$, the $\mathrm{C}$ - and R-maps produce only two other systems, namely those specified by the Lagrangians $\mathcal{L}_{C}$ and $\mathcal{L}_{R}$, the former being a classical topological field theory and the latter a reparametrisation invariant one. These three Lagrangians belong to a triangular set of fields theories which is closed under the action of the C- and R-maps and their inverse maps. The C-map acting on all three Lagrangians produces $\mathcal{L}_{C}$. The R-map acting on all three Lagrangians produces $\mathcal{L}_{R}$. And finally, the Lagrangian $\mathcal{L}$ is obtained from $\mathcal{L}_{C}$ and from $\mathcal{L}_{R}$ through the action of the inverse $\mathrm{C}$ - and R-maps respectively. This triangular relationship is the precise expression of the duality existing between classical topological field theories and reparametrisation invariant field theories mentioned in the introduction.

\section{The Generic Hierarchy}

Consider now a collection $F_{n}\left(\phi_{i}\right)(n=1,2, \ldots)$ of arbitrary functions of the first derivatives of a field $\phi\left(x_{i}\right)$ dependent on $d$ coordinates $x_{i}$. The associated Euler hierarchy of Lagrangians is then defined recursively by

$$
\mathcal{L}_{n}=F_{n} W_{n-1}, \quad W_{0}=1,
$$

where the $W_{n}(n=1,2, \ldots)$ are the equations of motion

$$
W_{n}=\mathcal{E} \mathcal{L}_{n},
$$


and $\mathcal{E}$ is the Euler operator defined in (2.2).

This describes the construction of the generic finite Euler hierarchy whose properties where discussed in the introduction. This section provides proofs for those properties. The fundamental result is the following identity

$$
W_{n}=n ! \operatorname{Tr}^{(n)}\left(A^{(1)}, \cdots, A^{(n)}\right),
$$

where

$$
A_{i j}^{(p)}=M_{i k}^{(p)} \phi_{k j}, \quad M_{i j}^{(p)}=\frac{\partial^{2} F_{p}}{\partial \phi_{i} \partial \phi_{j}}, \quad p=1,2, \cdots, n,
$$

and the function $\operatorname{Tr}^{(n)}$ in (5.3) is the generalised determinant defined in (A.5) in the Appendix. In fact, explicit expressions for $W_{n}$ are

$$
\begin{aligned}
W_{n} & =T_{i_{1} \cdots i_{n} ; j_{1} \cdots j_{n}}^{(n)} A_{i_{1} j_{1}}^{(1)} \cdots A_{i_{n} j_{n}}^{(n)} \\
& =\frac{1}{(d-n) !} \epsilon_{i_{1} \cdots i_{d}} \epsilon_{j_{1} \cdots j_{d}} A_{i_{1} j_{1}}^{(1)} \cdots A_{i_{n} j_{n}}^{(n)} \delta_{i_{n+1} j_{n+1}} \cdots \delta_{i_{d} j_{d}} \\
& =\frac{1}{(d-n) !} \epsilon_{i_{1} \cdots i_{d}} \epsilon_{j_{1} \cdots j_{d}} M_{i_{1} k_{1}}^{(1)} \cdots M_{i_{n} k_{n}}^{(n)} \phi_{k_{1} j_{1}} \cdots \phi_{k_{n} j_{n}} \delta_{i_{n+1} j_{n+1}} \cdots \delta_{i_{d} j_{d}} .
\end{aligned}
$$

$\left(T_{i_{1} \cdots i_{n} ; j_{1} \cdots j_{n}}^{(n)}\right.$ is defined in (A.1)).

Before coming to the consequences of this result, let us first discuss its proof, which uses some of the identities given in the Appendix and proceeds by induction. Obviously, (5.3) is true for $n=0$ by definition of $T^{(0)}$. The explicit calculation of $W_{1}$ gives

$$
\begin{aligned}
W_{1} & =\mathcal{E} \mathcal{L}_{1}=\mathcal{E}\left[F_{1}\left(\phi_{i}\right) W_{0}\right] \\
& =M_{i j}^{(1)} \phi_{i j} \\
& =\operatorname{tr} A^{(1)}=\operatorname{Tr}^{(1)}\left(A^{(1)}\right),
\end{aligned}
$$

in agreement with (5.3) (the last step uses (A.6)). Let us now assume that (5.3) holds for the first $n$ levels $(\ell=1,2, \cdots, n)$ of the hierarchy. We then have to show that at the next level $(\ell=n+1)$ the equation of motion $W_{n+1}$ computed from (5.2) does again agree with (5.3). The latter calculation separates according to the following identity

$$
\begin{aligned}
\mathcal{E}\left[W\left(\phi_{i}, \phi_{i j}\right) F\left(\phi_{i}\right)\right]= & {[\mathcal{E} W] F+2\left[\frac{\partial W}{\partial \phi_{i}}-\frac{1}{2} \partial_{j}\left(\frac{\partial W}{\partial \phi_{i j}}+\frac{\partial W}{\partial \phi_{j i}}\right)\right] \phi_{i k} \frac{\partial F}{\partial \phi_{k}}+} \\
& +\left[W \frac{\partial^{2} F}{\partial \phi_{i} \partial \phi_{j}} \phi_{i j}-\frac{\partial W}{\partial \phi_{i j}} \frac{\partial^{2} F}{\partial \phi_{k} \partial \phi_{l}} \phi_{i k} \phi_{j l}\right]
\end{aligned}
$$

which is thus used here with $\left(W=W_{n}\right)$ given in (5.5) and $\left(F=F_{n+1}\right)$. Since $W_{n}$ is the equation of motion for the Lagrangian $\left(\mathcal{L}_{n}=F_{n} W_{n-1}\right)$ which does not depend explicitly 
on the field $\phi, W_{n}$ is simply a surface term. Consequently ${ }^{[9,1]}$ its equation of motion $\left(\mathcal{E} W_{n}\right)$ - the first term in (5.7) - vanishes identically. In fact, we have

$$
\begin{aligned}
\mathcal{E} W_{n} & =\partial_{i}\left[\frac{\partial W_{n}}{\partial \phi_{i}}-\partial_{j} \frac{\partial W_{n}}{\partial \phi_{i j}}\right] \\
& =\partial_{i}\left[\frac{\partial W_{n}}{\partial \phi_{i}}-\frac{1}{2} \partial_{j}\left(\frac{\partial W_{n}}{\partial \phi_{i j}}+\frac{\partial W_{n}}{\partial \phi_{j i}}\right)\right],
\end{aligned}
$$

so that the equation of motion $\left(\mathcal{E} W_{n}\right)$ is also the divergence of one of the factors in the second term in (5.7). Actually, this factor itself vanishes identically, as we now discuss.

First, consider the terms

$$
\begin{aligned}
\partial_{j} & {\left[\frac{\partial W_{n}}{\partial \phi_{i j}}+\frac{\partial W_{n}}{\partial \phi_{j i}}\right]=} \\
& =\left[\frac{\partial^{2} W_{n}}{\partial \phi_{i j} \phi_{k}}+\frac{\partial^{2} W_{n}}{\partial \phi_{j i} \phi_{k}}\right] \phi_{j k}+\left[\frac{\partial^{2} W_{n}}{\partial \phi_{i j} \partial \phi_{k l}}+\frac{\partial^{2} W_{n}}{\partial \phi_{j i} \partial \phi_{k l}}\right] \phi_{j k l} .
\end{aligned}
$$

Using the fact that $W_{n}$ is symmetric in its arguments $M_{i j}^{(p)}(p=1,2, \cdots, n)$ and that the symbol $\epsilon_{i_{1} \cdots i_{d}}$ is fully antisymmetric, an explicit calculation shows that the last two terms in (5.9) vanish:

$$
\frac{\partial^{2} W_{n}}{\partial \phi_{i j} \partial \phi_{k l}} \phi_{j k l}=0, \quad \frac{\partial^{2} W_{n}}{\partial \phi_{j i} \partial \phi_{k l}} \phi_{j k l}=0 .
$$

Similarly, using the same properties and the fact that $\partial M_{i j}^{(p)} / \partial \phi_{k}$ is actually symmetric in all three indices $i, j, k$, an explicit analysis shows that the first two terms in (5.9) lead to

$$
\frac{\partial^{2} W_{n}}{\partial \phi_{i j} \partial \phi_{k}} \phi_{j k}=\frac{\partial W_{n}}{\partial \phi_{i}}, \quad \frac{\partial^{2} W_{n}}{\partial \phi_{j i} \partial \phi_{k}} \phi_{j k}=\frac{\partial W_{n}}{\partial \phi_{i}} .
$$

From the two results (5.10) and (5.11), it thus follows that we have indeed

$$
\frac{\partial W_{n}}{\partial \phi_{i}}-\frac{1}{2} \partial_{j}\left[\frac{\partial W_{n}}{\partial \phi_{i j}}+\frac{\partial W_{n}}{\partial \phi_{j i}}\right]=0 .
$$

Therefore, using (5.7), (5.8) and (5.12), the calculation of $W_{n+1}$ reduces to

$$
W_{n+1}=W_{n} M_{i j}^{(n+1)} \phi_{i j}-\frac{\partial W_{n}}{\partial \phi_{i j}} M_{k l}^{(n+1)} \phi_{i k} \phi_{j l} .
$$

However, it should be clear that the net effect of the last term in this relation is to replace each occurence of $\left(A_{i j}^{(p)}=M_{i k}^{(p)} \phi_{k j}\right)(p=1,2, \cdots, n)$ in $W_{n}$ with $\left(A_{i k}^{(p)} A_{k j}^{(n+1)}\right)$, namely

$$
\begin{aligned}
W_{n+1}= & T_{i_{1} \cdots i_{n} ; j_{1} \cdots j_{n}}^{(n)} A_{i_{1} j_{1}}^{(1)} \cdots A_{i_{n} j_{n}}^{(n)} A_{k k}^{(n+1)}- \\
& -\sum_{p=1}^{n} T_{i_{1} \cdots i_{n} ; j_{1} \cdots j_{n}}^{(n)} A_{i_{1} j_{1}}^{(1)} \cdots A_{i_{p-1} j_{p-1}}^{(p-1)} A_{i_{p} k}^{(p)} A_{k j_{p}}^{(n+1)} A_{i_{p+1} j_{p+1}}^{(p+1)} \cdots A_{i_{n} j_{n}}^{(n)} \\
= & {\left[T_{i_{1} \cdots i_{n} ; j_{1} \cdots j_{n}}^{(n)} \delta_{i_{n+1} j_{n+1}}\right.} \\
& \left.-\sum_{p=1}^{n} T_{i_{1} \cdots i_{n} ; j_{1} \cdots j_{p-1} j_{n+1} j_{p+1} \cdots j_{n}}^{(n)} \delta_{i_{n+1} j_{p}}\right] A_{i_{1} j_{1}}^{(1)} \cdots A_{i_{n+1} j_{n+1}}^{(n+1)} .
\end{aligned}
$$


Using now the identity (A.3), one finally obtains

$$
\begin{aligned}
W_{n+1} & =T_{i_{1} \cdots i_{n+1} ; j_{1} \cdots j_{n+1}}^{(n+1)} A_{i_{1} j_{1}}^{(1)} \cdots A_{i_{n+1} j_{n+1}}^{(n+1)} \\
& =(n+1) ! \operatorname{Tr}^{(n+1)}\left(A^{(1)}, \cdots, A^{(n+1)}\right)
\end{aligned}
$$

in agreement with (5.3). The iterative proof is thereby completed, and the main theorem of this paper established.

Given the result (5.3), let us consider its consequences. It is clear that $W_{n}$ is symmetric in the arguments $A^{(p)}$ or $M^{(p)}(p=1,2, \cdots, n)$. Namely, the order in which the multiplicative factors $F_{n}\left(\phi_{i}\right)$ are introduced in the calculation of $W_{n}$ is irrelevant; $W_{n}$ is fully symmetric in these functions. Moreover, according to the multiplication theorem (A.16), the dependence of $W_{n}$ on these functions and on the second derivatives $\phi_{i j}$ separates as

$$
W_{n}=n ! L_{i_{n+1} \cdots i_{d} ; j_{n+1} \cdots j_{d}}^{(n)}\left(M^{(1)}, \cdots, M^{(n)}\right) L_{j_{n+1} \cdots j_{d} ; i_{n+1} \cdots i_{d}}^{(n)}\left(\phi_{i j}\right)
$$

In particular, at level $(\ell=d)$ we obtain

$$
W_{d}=d ! \operatorname{Tr}^{(d)}\left(M^{(1)}, \cdots, M^{(d)}\right) \operatorname{det} \phi_{i j}
$$

showing that the dependence on the functions $F_{n}\left(\phi_{i}\right)(n=1,2, \cdots, d)$ factorizes at this level, leading to the following universal equation of motion for the Lagrangian $\mathcal{L}_{d}$ independently of the factors $F_{n}\left(\phi_{i}\right)(n=1,2, \cdots, d)$

$$
\operatorname{det} \phi_{i j}=0
$$

Since the choice for the functions $F_{n}\left(\phi_{i}\right)(n=1,2, \cdots, d)$ is totally arbitrary, (5.18) is indeed an example of an equation of motion admitting an infinite number of inequivalent Lagrangians. Moreover, since $W_{d}$ is always a surface term - being the equation of motion for a Lagrangian without an explicit dependence on the field $\phi-$, one also concludes that there is an infinite number of inequivalent conserved currents for the universal equation (5.18), suggesting its possible integrability.

The result (5.17) also implies that the hierarchy terminates at that level. Indeed, given any Lagrangian of the form

$$
\mathcal{L}\left(\phi_{i}, \phi_{i j}\right)=F\left(\phi_{i}\right) \operatorname{det} \phi_{i j}
$$

as is $\mathcal{L}_{d+1}$, it is easily shown using $(5.7)$ that its equation of motion $(\mathcal{E} \mathcal{L})$ vanishes identically. Hence, the universal equation (5.18) is also the last non trivial equation of motion for the hierarchy; the recursive construction in (5.1) and (5.2) always leads to

$$
W_{n}=0, \quad \text { for } n \geq d+1,
$$

which of course agrees implicitly with (5.3) since the functions $\operatorname{Tr}^{(n)}$ are defined only for $(n \leq d)$. 
In conclusion, we have established that the generic hierarchy indeed satisfies all the properties i), iib), iii) - v) described in the introduction, producing the universal equation (5.18) or (1.1) at level $(\ell=d)$. The generic hierarchy is thus an example of a finite Euler hierarchy with a universal equation of motion at the last level. In fact, in order for the hierarchy to terminate after a finite number of iterations and to lead to a universal equation of motion at the last level, it is crucial that the factors $F_{n}\left(\phi_{i}\right)$ be functions only of the first derivatives of the field $\phi$. Were these functions to also depend on the field itself, or on its second derivatives, the above properties would not follow. Namely, at each successive level of the iterative procedure, derivatives of the field of higher and higher order would be produced, never leading to a finite Euler hierarchy nor to a universal equation of motion. It is quite remarkable indeed that simply by taking for the multiplicative factors $F_{n}$ totally arbitrary functions of first derivatives only, the Euler hierarchy only involves first and second derivatives of the field, and terminates after a finite number of steps with a universal equation of motion which is moreover most probably an integrable system.

The above results generalise those of Ref.[1] corresponding to a situation where all functions $F_{n}\left(\phi_{i}\right)$ are taken to be identical. Specifically, consider the iterative procedure defining the generic hierarchy, with the choice

$$
F_{n}\left(\phi_{i}\right)=F_{0}\left(\phi_{i}\right), \quad n=1,2, \ldots,
$$

where $F_{0}\left(\phi_{i}\right)$ is totally arbitrary. Then from (5.5) and (5.20), it easily follows that

$$
\sum_{n=0}^{\infty} \frac{1}{n !} \lambda^{n} W_{n}=\operatorname{det}\left[\delta_{i j}+\lambda A_{i j}^{(0)}\right],
$$

with of course

$$
A_{i j}^{(0)}=M_{i k}^{(0)} \phi_{k j}, \quad M_{i j}^{(0)}=\frac{\partial^{2} F_{0}}{\partial \phi_{i} \partial \phi_{j}} .
$$

The result (5.22) is essentially one of the two main theorems of Ref.[1], which the present paper thus generalises.

To conclude this section, let us discuss the generalisation of the second main theorem of Ref.[1] leading to the Bateman hierarchy. Namely, consider again the above iterative procedure defining the generic hierarchy but now with factors $F_{n}\left(\phi_{i}\right)$ which are homogeneous functions of weight $(\alpha=1)$ but are otherwise arbitrary. This situation corresponds to the Bateman hierarchy. Thus, we now also have

$$
F_{n}\left(\lambda \phi_{i}\right)=\lambda F_{n}\left(\phi_{i}\right)
$$

leading to the further properties

$$
\begin{gathered}
M_{i j}^{(n)}\left(\lambda \phi_{i}\right)=\lambda^{-1} M_{i j}^{(n)}\left(\phi_{i}\right), \\
M_{i j}^{(n)}\left(\phi_{i}\right) \phi_{j}=0 \quad \Rightarrow \quad \operatorname{det} M_{i j}^{(n)}=0 .
\end{gathered}
$$


As we know from sect.2, the equations of motion $\left(\mathcal{E} F_{n}\right)$ are then invariant under arbitrary field redefinitions of $\phi$. Actually, this general covariance property then extends to the whole hierarchy. Indeed, consider an arbitrary transformation

$$
\varphi=F(\phi)
$$

so that

$$
\varphi_{i}=F^{\prime}(\phi) \phi_{i}, \quad \varphi_{i j}=F^{\prime}(\phi) \phi_{i j}+F^{\prime \prime}(\phi) \phi_{i} \phi_{j}
$$

It then follows that

$$
\begin{aligned}
A_{i j}^{(n)}\left(\varphi_{i}, \varphi_{i j}\right) & =M_{i k}^{(n)}\left(\varphi_{i}\right) \varphi_{k j} \\
& =F^{\prime}(\phi)^{-1} M_{i k}^{(n)}\left(\phi_{i}\right)\left[F^{\prime}(\phi) \phi_{k j}+F^{\prime \prime}(\phi) \phi_{k} \phi_{j}\right] \\
& =A_{i j}^{(n)}\left(\phi_{i}, \phi_{i j}\right)
\end{aligned}
$$

where the second line uses $(5.25 \mathrm{~b})$. Consequently, we have that

$$
W_{n}\left(\varphi_{i}, \varphi_{i j}\right)=W_{n}\left(\phi_{i}, \phi_{i j}\right)
$$

showing that all equations of motion of the hierarchy are then also invariant under arbitrary field redefinitions. In other words, all Lagrangians $\mathcal{L}_{n}$ constructed from the hierarchy define classical topological field theories of one field in $d$ dimensions, provided the multiplicative factors $F_{n}\left(\phi_{i}\right)$ themselves define such theories, namely are weight one homogeneous functions of $\phi_{i}$. In fact, all Lagrangians $\mathcal{L}_{n}$ constructed from the generic hierarchy then obey the homogeneity property (2.9) with weight $(\alpha=1)$. The Bateman hierarchy is thus a hierarchy of classical topological field theories.

More specifically, the homogeneous functions $F_{n}\left(\phi_{i}\right)$ may always be represented as

$$
F_{n}\left(\phi_{i}\right)=\phi_{d} G^{(n)}\left(\frac{\phi_{\alpha}}{\phi_{d}}\right)
$$

by an appropriate choice of labelling of the coordinates $x_{i}$. Here, the quantities $G^{(n)}$ are arbitrary functions of their $(d-1)$ arguments and $(\alpha, \beta)$ stand for the indices labelling the first $(d-1)$ coordinates among the coordinates $x_{i}$. Given (5.29), it then follows that

$$
M_{i j}^{(n)}\left(\phi_{i}\right)=\left(\begin{array}{cc}
\frac{G_{\alpha \beta}^{(n)}}{\phi_{d}} & \frac{G_{\alpha \beta}^{(n)}}{\phi_{d}}\left(-\frac{\phi_{\beta}}{\phi_{d}}\right) \\
\left(-\frac{\phi_{\alpha}}{\phi_{d}}\right) \frac{G_{\alpha \beta}^{(n)}}{\phi_{d}} & \left(-\frac{\phi_{\alpha}}{\phi_{d}}\right) \frac{G_{\alpha \beta}^{(n)}}{\phi_{d}}\left(-\frac{\phi_{\beta}}{\phi_{d}}\right)
\end{array}\right) .
$$

Here, $G_{\alpha \beta}^{(n)}$ is the matrix of second derivatives of the function $G^{(n)}$ with respect to its arguments - these arguments taking the values $\left(\phi_{\alpha} / \phi_{d}\right)$ in $(5.30)-$, and $(i, \alpha)$ and $(j, \beta)$ are line and column indices respectively. Using the degeneracy theorem (A.19) in the Appendix, one then concludes that the generalised determinant function $\operatorname{Tr}^{(d)}\left(M^{(1)}, \cdots, M^{(d)}\right)$ vanishes identically, implying that

$$
W_{d}\left(\phi_{i}, \phi_{i j}\right)=0
$$


In other words, the Bateman hierarchy terminates one step earlier than the generic hierarchy, namely at level $(\ell=d-1)$. The last non trivial equation of motion of the Bateman hierarchy is thus $W_{d-1}$. From (5.16) and the results (A.13) and (A.25), one finds explicitly

$$
W_{d-1}=-\frac{(d-1) !}{\phi_{d}^{d+1}} \operatorname{Tr}_{(d-1)}^{(d-1)}\left(G_{\alpha \beta}^{(1)}, \cdots, G_{\alpha \beta}^{(d-1)}\right) \operatorname{det}\left(\begin{array}{cc}
0 & \phi_{j} \\
\phi_{i} & \phi_{i j}
\end{array}\right) \text {. }
$$

Hence here again, the dependence on the choice of functions $F_{n}$ for the last non trivial equation of motion of the Bateman hierarchy factorizes out, leading to the universal Bateman equation of motion at level $(\ell=d-1)$

$$
\operatorname{det}\left(\begin{array}{cc}
0 & \phi_{j} \\
\phi_{i} & \phi_{i j}
\end{array}\right)=0
$$

These are thus the results promised in the introduction in the case of the Bateman hierarchy. They also generalise the conclusions of Ref.[1] which were obtained in the case when all homogeneous weight one functions $F_{n}$ are equal. Note that any field configuration obeying the relation

$$
\phi_{i j}=S_{i} \phi_{j}+S_{j} \phi_{i}
$$

for some coefficients $S_{i}$, is always a solution to all equations of motion of the Bateman hierarchy - generalising a similar statement in Ref.[1]. Indeed, when (5.34) is used in the expression for $W_{n}$, each of the factors $\phi_{i}$ is always contracted with one of the matrices $M_{i j}^{(p)}(p=1,2, \cdots, n)$, leading to a vanishing contribution since $\phi_{i}$ is a zero mode of these matrices (see $(5.25 \mathrm{~b}))$. In particular, configurations $\phi\left(x_{i}\right)$ implicitly defined by the equation

$$
x_{i} F_{i}(\phi)=c
$$

with $F_{i}(\phi)$ being arbitrary functions and $c$ an arbitrary constant, always obey the identity (5.34). Thus, (5.35) defines $^{[1]}$ implicit solutions to all the equations of motion of the Bateman hierarchy, including the Bateman equation (5.33).

Finally, one may wonder whether by imposing further conditions on the functions $F_{n}\left(\phi_{i}\right)$, the Bateman hierarchy itself would not terminate a step earlier in the same way that the generic hierarchy terminates at level $(\ell=d-1)$ when the functions $F_{n}\left(\phi_{i}\right)$ are homogeneous and of weight one. Actually, the explicit result (5.32) shows that $W_{d-1}$ itself vanishes whenever the generalised determinant which is the prefactor in that expression vanishes. From the discussion around (A.19) in the Appendix, it follows that such a situation indeed occurs provided the functions $G^{(n)}$ introduced in (5.29) are themselves homogeneous functions of weight one, namely

$$
G^{(n)}\left(t_{\alpha}\right)=t_{d-1} H^{(n)}\left(\frac{t_{1}}{t_{d-1}}, \cdots, \frac{t_{d-2}}{t_{d-1}}\right)
$$

However, this choice implies

$$
F_{n}\left(\phi_{i}\right)=\phi_{d-1} H^{(n)}\left(\frac{\phi_{1}}{\phi_{d-1}}, \cdots, \frac{\phi_{d-2}}{\phi_{d-1}}\right),
$$


thus showing that one is then actually dealing with a Bateman hierarchy for a single field in $(d-1)$ dimensions. The last coordinate $x_{d}$ plays no rôle in the dynamical evolution of the field theory, since the functions $F_{n}$, hence also the successive Lagrangians in the hierarchy, are independent of derivatives of the field with respect to that coordinate. Therefore, weight one homogeneity is the only possible restriction on the functions $F_{n}$ leading to a truncation of the generic hierarchy at an earlier stage. Correspondingly, one obtains the Bateman hierarchy with the universal Bateman equation (5.33) at the last non trivial level.

\section{Finite Euler Hierarchies for Many Fields}

Given the generic finite Euler hierarchy for one field with a universal equation of motion at the last level, it is now possible to construct other such hierarchies for one or more fields. One obvious way is to apply the $\mathrm{C}$ - or R-maps to the generic hierarchy for one field depending on $d$ coordinates. Using the C-map, one obtains a finite Euler hierarchy of classical topological field theories for one field in $(d+1)$ dimensions. Actually, this hierarchy is precisely the Bateman hierarchy described at the end of the previous section, with the Bateman equation (5.33) as its universal equation at level $(\ell=d)$. Using the R-map, one obtains ${ }^{[2]}$ a finite Euler hierarchy of reparametrisation invariant field theories for $(d+1)$ fields in $d$ dimensions, whose universal equation at level $(\ell=d)$ was given in (1.3) (there are of course $(d+1)$ equations of motion at each level of this hierarchy, but the Ward identities of reparametrisation invariance imply that there is actually only one independent equation at each level. At the last level, this is the universal equation (1.3)).

However, it is possible to consider a somewhat wider framework which includes the hierarchies just mentioned as particular cases, and allows to have more than one field still in the context of the generic hierarchy. The idea is simply to consider the generic hierarchy with the original field $\phi\left(x_{i}\right)$ now being actually a linear combination of a collection of fields $\phi^{a}\left(x_{i}\right)$, namely $\left(\phi\left(x_{i}\right)=\phi^{a}\left(x_{i}\right) \lambda^{a}\right)$. The coefficients $\lambda^{a}$ are totally arbitrary, and it is assumed that whatever the equations of motion, they are expanded in terms of these coefficients and have to hold independently of their values. The situation discussed above is recovered simply by setting the then single coefficient $(\lambda=1)$, namely by absorbing it in the normalisation of the field $\phi$ itself. For this reason, the hierarchies described in the previous paragraph are not discussed separately here.

First, let us thus discuss the generic case of $D$ fields $\phi^{a}\left(x_{i}\right)$ functions of $d$ coordinates

$x_{i}$. Consider a collection of arbitrary functions $F_{n}\left(t_{i}\right)(n=1,2, \ldots)$ of $d$ arguments denoted $t_{i}$ for our present purpose, with the symmetric matrix functions

$$
M_{i j}^{(n)}\left(t_{i}\right)=\frac{\partial^{2} F_{n}}{\partial t_{i} \partial t_{j}}\left(t_{i}\right) .
$$

Define now the iterative construction of the following Euler hierarchy. Its Lagrangians for $(n=1,2, \ldots)$ are given by

$$
\mathcal{L}_{\left(n ; a_{1} \cdots a_{n-1}\right)}\left(\phi_{i}^{a}, \phi_{i j}^{a} ; \lambda^{a}\right)=F_{n}\left(\phi_{i}^{a} \lambda^{a}\right) W_{\left(n-1 ; a_{1} \cdots a_{n-1}\right)}\left(\phi_{i}^{a}, \phi_{i j}^{a} ; \lambda^{a}\right)
$$


where

$$
W_{0}=1
$$

and

$$
W_{\left(n ; a_{1} \cdots a_{n}\right)}\left(\phi_{i}^{a}, \phi_{i j}^{a} ; \lambda^{a}\right)=\mathcal{E}_{\phi^{a_{n}}} \mathcal{L}_{\left(n ; a_{1} \cdots a_{n-1}\right)}\left[\phi^{a}\right], \quad \text { for } n=1,2, \ldots
$$

By relating this construction to that of the generic hierarchy for the field $\left(\Phi=\phi^{a} \lambda^{a}\right)$ with the functions $F_{n}\left(\Phi_{i}\right)$, it follows that we actually have the explicit result

$$
W_{\left(n ; a_{1} \cdots a_{n}\right)}\left(\phi_{i}^{a}, \phi_{i j}^{a} ; \lambda^{a}\right)=n ! \lambda^{a_{1}} \cdots \lambda^{a_{n}} \operatorname{Tr}^{(n)}\left(A^{(1)}, \cdots, A^{(n)}\right)
$$

for $(n=0,1, \ldots)$, with

$$
A_{i j}^{(p)}=M_{i k}^{(p)}\left(\phi_{i}^{a} \lambda^{a}\right) \phi_{k j}^{a} \lambda^{a}, \quad p=1,2, \cdots, n
$$

In particular, this implies that the hierarchy defined in (6.2) terminates at level $(\ell=d)$, with the universal equations

$$
\operatorname{det}\left(\phi_{i j}^{a} \lambda^{a}\right)=0
$$

As before, the dependence of the last non trivial equations of motion $W_{\left(d ; a_{1} \cdots a_{d}\right)}$ on the functions $F_{n}$ factors out, leaving (6.5) as the universal equations for this hierarchy. By expansion in the coefficients $\lambda^{a}$, one thus obtains $\left(\begin{array}{c}d+D-1 \\ D-1\end{array}\right)$ equations of motion for the $D$ fields $\phi^{a}$. Even though this may seem to be an overdetermined system - except when $(D=1)$ or $(d=1)-$, it is easy to see that it nevertheless possesses non trivial solutions (some are discussed in the next section). Moreover, note that there is essentially only one independent Lagrangian at every level of the hierarchy, since the free indices $a_{k}$ attached to the Lagrangians (6.2a) are simply carried by the coefficients $\lambda^{a_{k}}$ as overall factors.

Obviously, the generic hierarchy is indeed recovered from the expressions above in the case $(D=1)$ by setting $(\lambda=1)$. The other situation were the number of universal equations of motion is equal to the number of fields, namely $(d=1)$, corresponds to a hierarchy terminating after one step with the equations

$$
\phi_{x x}^{a}=0, \quad a=1,2, \cdots, D
$$

The general solution to these equations is obvious.

It is straightforward to apply the C-map to the generic hierarchy for many fields defined above. As we know, this leads to a hierarchy of classical topological field theories, which is constructed as follows (the notation is that of sect.3). Consider $(D=p)$ fields $\phi^{a}\left(x_{i}\right)$ dependent on $(d=p+q)$ coordinates $\left(x_{i}=\left(x_{\alpha}, y^{a}\right)\right)$ (there are thus $q$ coordinates $x_{\alpha}$ and $p$ coordinates $\left.y^{a}\right)$, and introduce the matrix

$$
M_{a}^{b}=\frac{\partial \phi^{b}}{\partial y^{a}}
$$


Consider now functions $F_{n}(n=1,2, \ldots)$ given by

$$
F_{n}\left(\phi_{i}^{a} ; \lambda^{a}\right)=\left(\operatorname{det} M_{a}^{b}\right) G_{n}\left(-\frac{\partial \phi^{a}}{\partial x_{\alpha}}\left(M^{-1}\right)_{a}{ }^{b} \lambda^{b}\right),
$$

where $G_{n}\left(t_{\alpha}\right)$ are arbitrary functions of $q$ arguments $t_{\alpha}$, with the symmetric matrix functions

$$
M_{\alpha \beta}^{(n)}\left(t_{\alpha}\right)=\frac{\partial^{2} G_{n}}{\partial t_{\alpha} \partial t_{\beta}}\left(t_{\alpha}\right) .
$$

Define then iteratively the following Euler hierarchy. Its Lagrangians for $(n=1,2, \ldots)$ are given by

$$
\begin{aligned}
& \mathcal{L}_{\left(n ; a_{1} \cdots a_{n-1}\right)}\left(\phi_{i}^{a}, \phi_{i j}^{a} ; \lambda^{a}\right)= \\
& \quad=F_{n}\left(\phi_{i}^{a} ; \lambda^{a}\right)\left(\operatorname{det} M_{a}{ }^{b}\right)^{-1} M_{a_{n-1}}{ }^{b} W_{\left(n-1 ; a_{1} \cdots a_{n-2} b\right)}\left(\phi_{i}^{a}, \phi_{i j}^{a} ; \lambda^{a}\right),
\end{aligned}
$$

where

$$
W_{0}=\operatorname{det} M_{a}^{b}
$$

and

$$
W_{\left(n ; a_{1} \cdots a_{n}\right)}\left(\phi_{i}^{a}, \phi_{i j}^{a} ; \lambda^{a}\right)=\mathcal{E}_{\phi^{a_{n}}} \mathcal{L}_{\left(n ; a_{1} \cdots a_{n-1}\right)}\left[\phi^{a}\right], \quad \text { for } n=0,1, \ldots
$$

From the C-map applied to the hierarchy defined in (6.2), it follows that we have in fact for $(n=0,1, \ldots)$

$$
\begin{aligned}
& W_{\left(n ; a_{1} \cdots a_{n}\right)}\left(\phi_{i}^{a}, \phi_{i j}^{a} ; \lambda^{a}\right)= \\
& \quad=(-1)^{n} n !\left(\operatorname{det} M_{a}{ }^{b}\right) \lambda^{a_{1}} \cdots \lambda^{a_{n-1}}\left(M^{-1}\right) a_{n}{ }^{b} \lambda^{b} \operatorname{Tr}^{(n)}\left(A^{(1)}, \cdots, A^{(n)}\right),
\end{aligned}
$$

with

$$
A_{\alpha \beta}^{(p)}=M_{\alpha \gamma}^{(p)}\left(Y_{\alpha}^{a} \lambda^{a}\right) Y_{\gamma \beta}^{a} \lambda^{a},
$$

where the quantities $Y_{\alpha}^{a}$ and $Y_{\alpha \beta}^{a}$ are given in (3.8) and (3.9). As already mentioned, the construction in (6.10) leads to a hierarchy of classical topological field theories. In particular, note how the functions $F_{n}$ in (6.8) obey the homogeneity property (2.9) with weight $(\alpha=1)$, ensuring that their equations of motion are generally covariant under arbitrary field redefinitions. The homogeneity property $(2.9)$ with weight $(\alpha=1)$ of course extends to the whole hierarchy itself. Also, note that there is in fact essentially only one independent Lagrangian at each level of the hierarchy.

From (6.11), it follows that the hierarchy (6.10) is again a finite one, terminating at the level $(\ell=q=d-D)$ with equations of motion which, up to a factor depending on the functions $F_{n}$, are universal and given by

$$
\operatorname{det}\left(Y_{\alpha \beta}^{a} \lambda^{a}\right)=0 .
$$


Using the identity

$$
\operatorname{det}\left(X_{\alpha \beta}-A_{\alpha a} B_{a \beta}-B_{a \alpha} A_{\beta a}+A_{\alpha a} Y_{a b} A_{\beta b}\right)=\operatorname{det}\left(\begin{array}{ccc}
0_{a b} & A_{\beta a} & \delta_{a b} \\
A_{\alpha b} & X_{\alpha \beta} & B_{b \alpha} \\
\delta_{a b} & B_{a \beta} & Y_{a b}
\end{array}\right)
$$

(here, $(a, \alpha)$ and $(b, \beta)$ are line and column indices respectively), the equations implied by (6.13) are equivalent to

$$
\operatorname{det}\left(\begin{array}{cc}
0 & \phi_{i}^{b} \\
\phi_{j}^{a} & \phi_{i j}^{c} \lambda^{c}
\end{array}\right)=0
$$

(here, $(i, a)$ and $(j, b)$ are line and column indices respectively). The hierarchy (6.10) thus leads to the generally covariant equations of motion (1.5) as universal equations of motion arising at level $(\ell=d-D)$. This establishes the conjecture of Ref.[1]. Clearly, the hierarchy (6.10) is a generalisation of the Bateman hierarchy for one field. The latter is indeed recovered when $(D=1=p)$ and setting $(\lambda=1)$ in the above expressions. Note how the functions $F_{n}$ in (6.8) are then precisely of the form (5.29) used in the construction of the Bateman hierarchy as a particular reduction of the generic hierarchy.

As pointed out in the introduction, the set of $\left(\begin{array}{c}d-1 \\ D-1\end{array}\right)$ equations is in general overdetermined, except when $(D=1)$ or $(D=d-1)$. These two special cases of course correspond through the C-map to the two distinguished cases $(D=1)$ and $(d=1)$ mentioned above in the context of the generic hierarchy for many fields. Here, the situation for $(D=1)$ is that of the Bateman hierarchy. On the other hand, the situation with $(D=d-1)$ corresponds precisely to universal equations already given in Ref.[1]. Namely, when $(D=d-1)$, the hierarchy (6.10) terminates after only one iteration with the universal equations of motion

$$
J_{i} J_{j} \phi_{i j}^{a}=0
$$

where the Jacobians $J_{i}$ are defined by

$$
\operatorname{det}\left(\phi_{i}^{b} \quad \zeta_{i}\right)=\zeta_{i} J_{i}
$$

(here, $i$ and $b$ are line and column indices respectively). The solutions to these equations are obtained from those of (6.6) through the C-map.

Finally, let us describe the result of the R-map applied to the generic hierarchy for more than one field defined in (6.2). As we know, one then obtains hierarchies of reparametrisation invariant field theories. Using the notations of sect.4, consider $(D=p+q)$ fields $\left(\phi^{a}\left(x_{j}\right)=\left(\phi^{\mu}\left(x_{j}\right), z_{i}\left(x_{j}\right)\right)\right)$ dependent on $(d=q)$ coordinates $x_{i}$ (there are thus $p$ fields $\phi^{\mu}$ and $q$ fields $z_{i}$ ), and define the matrix

$$
M_{i}^{j}=\frac{\partial z_{j}}{\partial x_{i}}
$$

Consider now a collection of functions $F_{n}(n=1,2, \ldots)$ given by

$$
F_{n}\left(\phi_{i}^{a} ; \lambda^{\mu}\right)=\left(\operatorname{det} M_{i}^{j}\right) G_{n}\left(\left(M^{-1}\right)_{i}{ }^{j} \frac{\partial \phi^{\mu}}{\partial x_{j}} \lambda^{\mu}\right),
$$


where $G_{n}\left(t_{i}\right)$ are arbitrary functions of $(d=q)$ arguments $t_{i}$, with the symmetric matrix functions

$$
M_{i j}^{(n)}\left(t_{i}\right)=\frac{\partial^{2} G_{n}}{\partial t_{i} \partial t_{j}}\left(t_{i}\right)
$$

Define then iteratively the following hierarchy. The Lagrangians for $(n=1,2, \ldots)$ are given by

$$
\mathcal{L}_{\left(n ; \mu_{1} \cdots \mu_{n-1}\right)}\left(\phi_{i}^{a}, \phi_{i j}^{a} ; \lambda^{\mu}\right)=F_{n}\left(\phi_{i}^{a} ; \lambda^{\mu}\right)\left(\operatorname{det} M_{i}^{j}\right)^{-1} W_{\left(n-1 ; \mu_{1} \cdots \mu_{n-1}\right)}\left(\phi_{i}^{a}, \phi_{i j}^{a} ; \lambda^{\mu}\right),
$$

where

$$
W_{0}=\operatorname{det} M_{i}^{j}
$$

and

$$
W_{\left(n ; \mu_{1} \cdots \mu_{n}\right)}\left(\phi_{i}^{a}, \phi_{i j}^{a} ; \lambda^{\mu}\right)=\mathcal{E}_{\phi^{\mu_{n}}} \mathcal{L}_{\left(n ; \mu_{1} \cdots \mu_{n-1}\right)}\left[\phi^{a}\right], \quad \text { for } n=0,1, \ldots
$$

From the R-map, one then finds the explicit representation for $(n=0,1,2, \ldots)$

$$
W_{\left(n ; \mu_{1} \cdots \mu_{n}\right)}\left(\phi_{i}^{a}, \phi_{i j}^{a} ; \lambda^{\mu}\right)=n !\left(\operatorname{det} M_{i}^{j}\right) \lambda^{\mu_{1}} \cdots \lambda^{\mu_{n}} \operatorname{Tr}^{(n)}\left(A^{(1)}, \cdots, A^{(n)}\right)
$$

where

$$
A_{i j}^{(p)}=M_{i k}^{(p)}\left(Y_{i}^{\mu} \lambda^{\mu}\right) Y_{k j}^{\mu} \lambda^{\mu}
$$

with $Y_{i}^{\mu}$ and $Y_{i j}^{\mu}$ given in (4.6) and (4.7). As already mentioned, the construction (6.21) leads to a Euler hierarchy of reparametrisation invariant field theories. In particular, the functions $F_{n}$ obey the homogeneity property (2.3) with weight $(\alpha=1)$, ensuring reparametrisation invariance of the field theories they define. The property (2.3) with weight $(\alpha=1)$ of course extends to the whole hierarchy. Note that the hierarchy is defined only in terms of equations of motion for the fields $\phi^{\mu}$. This is because the Ward identities of reparametrisation invariance relate the equations of motion for the fields $z_{i}$ to those for the fields $\phi^{\mu}$, the latter thus being the only independent ones. Also, note that once again there is essentially only one independent Lagrangian at each level of the hierarchy.

As before, (6.22) implies that the hierarchy (6.21) is also finite, and terminates at level $(\ell=d=q)$ with equations of motion which, up to a factor depending on the choice of functions $F_{n}$, are universal equations with reparametrisation invariance. These universal equations are obtained from

$$
\operatorname{det}\left(Y_{i j}^{\mu} \lambda^{\mu}\right)=0
$$

expanded in terms of the coefficients $\lambda^{\mu}$. For convenience, let us introduce Jacobians $J$ and $J_{i}^{\mu}$ generated by the function

$$
\operatorname{det}\left(\begin{array}{cc}
\zeta & \zeta^{j} \\
\phi_{i}^{\mu} \lambda^{\mu} & M_{i}^{j}
\end{array}\right)=\zeta J+\zeta^{i} J_{i}^{\mu} \lambda^{\mu},
$$

where as usual $i$ and $j$ denote line and column indices respectively. The explicit relations are

$$
J=\operatorname{det} M_{i}{ }^{j}, \quad J_{i}^{\mu}=-\left(\operatorname{det} M_{i}{ }^{j}\right)\left(M^{-1}\right)_{i}{ }^{j} \frac{\partial \phi^{\mu}}{\partial x_{j}} .
$$


Note that $J_{i}^{\mu}$ is the determinant of the matrix $M_{i}{ }^{j}$ in which the $i^{\text {th }}$ line is replaced by the line $\left(\partial \phi^{\mu} / \partial x_{j}\right)$. In terms of these definitions, the universal equations (6.24) reduce to

$$
\operatorname{det}\left[\left(J \frac{\partial^{2} \phi^{\mu}}{\partial x_{i} \partial x_{j}}+J_{k}^{\mu} \frac{\partial^{2} z_{k}}{\partial x_{i} \partial x_{j}}\right) \lambda^{\mu}\right]=0 .
$$

One thus obtains $\left(\left(\begin{array}{c}p+q-1 \\ q\end{array}\right)=\left(\begin{array}{c}D-1 \\ d\end{array}\right)\right)$ independent universal equations of motion for a system of $D$ fields which is reparametrisation invariant in $d$ coordinates. Except when $(D=d+1)$ or $(d=1)$ - corresponding through the R-map to the two cases when the universal equations of motion of the generic hierarchy (6.2) are not overdetermined -, the system of universal equations (6.27) is overdetermined since the number of independent equations should be $(D-d)$, when accounting for reparametrisation invariance. Nevertheless, it is easy to see that there exist non trivial solutions to these equations.

From the results above, the reparametrisation invariant hierarchy obtained from the R-map acting on the generic hierarchy for one field is recovered when $(D=d+1)$, i.e. $(p=1)$, and setting $(\lambda=1)$. The corresponding hierarchy then terminates at level $(\ell=d)$ with the universal reparametrisation invariant equation of motion (1.3) first considered in Ref.[2]. This is one of the two instances when the number of fields and universal equations are equal, up to the Ward identities of reparametrisation invariance. The other such instance is when $(d=1)$. In this case, the hierarchy (6.21) terminates after one iteration, with the $(D-1)$ independent universal equations of motion

$$
\frac{\partial z}{\partial x} \frac{\partial^{2} \phi^{\mu}}{\partial x^{2}}=\frac{\partial \phi^{\mu}}{\partial x} \frac{\partial^{2} z}{\partial x^{2}} .
$$

Consequently we then have ${ }^{[2]}$

$$
\frac{\partial \phi^{a}}{\partial x} \frac{\partial^{2} \phi^{b}}{\partial x^{2}}=\frac{\partial \phi^{b}}{\partial x} \frac{\partial^{2} \phi^{a}}{\partial x^{2}}, \text { for all } a, b,
$$

whose solutions are obtained from those of (6.6) through the R-map.

To conclude, let us emphasize the fact that the hierarchies constructed in this section are generalisations of the finite Euler hierarchies with universal equations discovered and conjectured in Refs.[1,2]. These generalisations now apply to field theories for which the difference between the numbers of fields and coordinates is totally arbitrary. The associated universal equations are given in (6.5), (6.15) and (6.27) and are dual to one another under the C- and R-maps. These equations include as particular examples the cases (1.1), (1.2) and (1.3) as well as (6.6), (6.16) and (6.29), corresponding to the finite Euler hierarchies considered previously ${ }^{[1,2]}$.

\section{Examples of Solutions}

As pointed out repeatedly, the universal equations of motion discussed above possess an infinite number of conservation laws. Indeed, these equations derive from an infinity 
of inequivalent Lagrangians, i.e. Lagrangians not differing by surface terms, which do not explicitly depend on the fields themselves but only on their derivatives. Hence, the corresponding equations of motion take the form

$$
\mathcal{E}_{a} \mathcal{L}\left(\phi_{i}^{a}, \phi_{i j}^{a}\right)=\partial_{i}\left[\frac{\partial \mathcal{L}}{\partial \phi_{i}^{a}}-\partial_{j} \frac{\partial \mathcal{L}}{\partial \phi_{i j}^{a}}\right]
$$

which are thus conservation laws for field configurations solving the equations of motion. This property strongly suggests the possible integrability of the universal equations, namely the existence of an infinite number of conserved charges in involution for some symplectic structure defining a Hamiltonian formulation for these systems.

This suggestion is also supported from a somewhat different point of view, although not completely unrelated to the previous remark. All theories in this paper are invariant under translations in the base space coordinates $x_{i}$. Hence, by Noether's first theorem ${ }^{[10]}$, there exists a conserved current associated to this symmetry: the stress-tensor $T_{i j}$. For Lagrangians $\mathcal{L}\left(\phi_{i}^{a}, \phi_{i j}^{a}\right)$ depending only on first and second derivatives of the fields, we have explicitly

$$
T_{i j}=\phi_{i}^{a} \frac{\partial \mathcal{L}}{\partial \phi_{j}^{a}}+\frac{1}{2} \phi_{i k}^{a}\left[\frac{\partial \mathcal{L}}{\partial \phi_{j k}^{a}}+\frac{\partial \mathcal{L}}{\partial \phi_{k j}^{a}}\right]-\frac{1}{2} \phi_{i}^{a} \partial_{k}\left[\frac{\partial \mathcal{L}}{\partial \phi_{j k}^{a}}+\frac{\partial \mathcal{L}}{\partial \phi_{k j}^{a}}\right]-\delta_{i j} \mathcal{L}
$$

(Note that for reparametrisation invariant theories obeying the homogeneity property $(2.3)$ with weight $(\alpha=1)$, this quantity vanishes identically). The conservation equation for the stress-tensor is

$$
\partial_{j} T_{i j}=\phi_{i}^{a} \mathcal{E}_{a} \mathcal{L}
$$

Therefore, associated to the infinity of Lagrangians leading to the universal equations of this paper, solutions to these equations possess the infinity of conservation laws for the corresponding stress-tensors $T_{i j}$ whenever the latter is non trivial.

It is not the purpose of this paper to address the issue of integrability, neither for cases 1) - 3) of the introduction nor for the more general universal equations of the previous section. However, we present in this section large classes of solutions, thereby providing circumstancial evidence that we are indeed dealing with integrable systems. In the simplest cases, it is possible to give the general solution to the equations - sometimes taking advantage of the $\mathrm{C}$ - and $\mathrm{R}$-maps -, thus implicitly proving their integrability. On the other hand, let us recall ${ }^{[1]}$ how the integrability of the two dimensional Bateman equation may be displayed explicitly. Introducing ${ }^{[5,1]}$ the variables

$$
u\left(x_{1}, x_{2}\right)=\frac{\phi_{1}\left(x_{1}, x_{2}\right)}{\phi_{2}\left(x_{1}, x_{2}\right)}
$$

the two dimensional Bateman equation (1.2) reduces to the following non linear equation for $u\left(x_{1}, x_{2}\right)$

$$
u_{1}=u u_{2}
$$


This is recognised as the KdV equation without the term in $u_{222}$. Obviously, all integer powers of $u$ define densities of conserved charges for this system, which are also in involution for either of the two known symplectic structures for the KdV hierarchy.

Let us first concentrate on the simplest cases for which the general solution may be constructed, in the case of the generic hierarchy for one field and its C- and R-dual representations. Obviously, the Bateman equation in one dimension is trivially solved: $\phi(x)$ is simply any constant. Next, consider the universal equation (1.1) for the generic hierarchy in one dimension, which is simply

$$
\phi_{x x}=0 .
$$

The general solution is thus

$$
\phi(x)=A x+B,
$$

where $A$ and $B$ are arbitrary constants. Using the R-map, we then directly obtain the general solution to the reparametrisation invariant equation (1.3) in the case of two fields $\phi^{1}$ and $\phi^{2}$ in one dimension. The solution is

$$
\phi^{1}(x)=A f(x)+B, \quad \phi^{2}(x)=f(x),
$$

where $f(x)$ is any function of the coordinate $x$, possibly constant, and $A$ and $B$ are arbitrary constants. Actually, the condition (4.4) - necessary for the application of the R-map requires the function $f(x)$ to be non constant. The only solutions to $(1.3)$ when $(d=1)$ which do not obey (4.4) are precisely those for which $f(x)$ is constant. Hence, (7.8) indeed defines the general solution to (1.3) in this case, with $f(x)$ being any function.

Using the C-map, (7.7) also leads to the following implicit solutions to the Bateman equation (1.2) in two dimensions

$$
x_{2}=A(\phi) x_{1}+B(\phi),
$$

where $A(\phi)$ and $B(\phi)$ are now arbitrary functions of $\phi$. The C-map does not necessarily provide all solutions however, since the non degeneracy condition (3.6) must be obeyed. In the present case, the solutions which are not given by (7.9) are simply those which are independent of $x_{2}$. Obviously, all solutions are obtained when (7.9) is extended to

$$
x_{1} F(\phi)+x_{2} G(\phi)+H(\phi)=0,
$$

where $F(\phi), G(\phi)$ and $H(\phi)$ are arbitrary functions. Any choice with $(G(\phi) \neq 0)$ leads back to (7.9), whereas taking $(G(\phi)=0)$ leads to all configurations $\phi\left(x_{1}\right)$ function of $x_{1}$ only, which are indeed always solutions.

The results above thus completely describe the general solutions to all three equations $(1.1)-(1.3)$ when $(d=1)$. These systems are clearly integrable, since one of the members of this "duality triangle" is the two dimensional Bateman equation, which is indeed an integrable system as was explained in (7.5). Note also how (7.10) is equivalent to (1.6) in this case, showing that (1.6) indeed defines the general solution to the Bateman equation in two dimensions. 
Let us now turn to the universal equation (1.1) of the generic hierarchy when $(d=2)$. This is a particular reduction of the Plebanski equation ${ }^{[3]}$ for self-dual gravity in four dimensions, which is known to be integrable. Therefore, its dual pair - the Bateman equation (1.2) in three dimensions and the universal string equation (1.3) in three spacetime dimensions - are also integrable systems. In fact, all solutions to these equations may be defined implicitly. Consider first the equation (1.1) in this case. Introducing the new variable $\left(h\left(x_{1}, x_{2}\right)=\phi_{2}\left(x_{1}, x_{2}\right)\right)$, it follows ${ }^{[3]}$ that if $\phi\left(x_{1}, x_{2}\right)$ solves $(1.1)$ then $h\left(x_{1}, x_{2}\right)$ is a solution to the two dimensional Bateman equation, whose general solutions are given above. Considering then separately the two cases when either $h\left(x_{1}, x_{2}\right)$ is independent of $x_{2}$ or is given as in (7.9), and using the fact that any function of a solution to the Bateman equation is again a solution, one concludes that all solutions to $(1.1)$ when $(d=2)$ are obtained from the following two relations

$$
\phi\left(x_{1}, x_{2}\right)=x_{1} F(h)+x_{2} G(h)+H(h),
$$

and

$$
x_{1} \frac{\mathrm{d} F}{\mathrm{~d} h}(h)+x_{2} \frac{\mathrm{d} G}{\mathrm{~d} h}(h)+\frac{\mathrm{d} H}{\mathrm{~d} h}(h)=0 .
$$

Namely, (7.12) defines an implicit solution for $h\left(x_{1}, x_{2}\right)$ which is then used in (7.11) to obtain the solution for $\phi\left(x_{1}, x_{2}\right)$. Here, $F(h), G(h)$ and $H(h)$ are totally arbitrary functions.

Using the R-map, one then also obtains the general solution to the universal string equation (1.3) in a three dimensional spacetime. The three fields $\phi^{a}\left(x_{1}, x_{2}\right)(a=1,2,3)$ functions of the coordinates $\left(x_{1}, x_{2}\right)$ are then given by

$$
\begin{aligned}
\phi^{1}\left(x_{1}, x_{2}\right) & =f_{1}\left(x_{1}, x_{2}\right) F(h)+f_{2}\left(x_{1}, x_{2}\right) G(h)+H(h), \\
\phi^{2}\left(x_{1}, x_{2}\right) & =f_{1}\left(x_{1}, x_{2}\right), \\
\phi^{3}\left(x_{1}, x_{2}\right) & =f_{2}\left(x_{1}, x_{2}\right),
\end{aligned}
$$

where $f_{1}\left(x_{1}, x_{2}\right)$ and $f_{2}\left(x_{1}, x_{2}\right)$ as well as $F(h), G(h)$ and $H(h)$ are arbitrary functions, and $h\left(x_{1}, x_{2}\right)$ is implicitly given by the relation

$$
f_{1}\left(x_{1}, x_{2}\right) \frac{\mathrm{d} F}{\mathrm{~d} h}(h)+f_{2}\left(x_{1}, x_{2}\right) \frac{\mathrm{d} G}{\mathrm{~d} h}(h)+\frac{\mathrm{d} H}{\mathrm{~d} h}(h)=0 .
$$

Here again, the non degeneracy condition (4.4) requires that the Jacobian $\left(\operatorname{det}\left(\partial f_{j} / \partial x_{i}\right)\right)$ is non vanishing, but it is easy to check that the solutions to (1.3) which do not obey this condition are included in (7.13) and (7.14).

Similarly, using the C-map, all solutions to the three dimensional Bateman equation (1.2) are obtained from the implicit definitions

$$
x_{1} F_{1}(h ; \phi)+x_{2} F_{2}(h ; \phi)+x_{3} F_{3}(h ; \phi)+H(h ; \phi)=0,
$$

and

$$
x_{1} \frac{\partial F_{1}}{\partial h}(h ; \phi)+x_{2} \frac{\partial F_{2}}{\partial h}(h ; \phi)+x_{3} \frac{\partial F_{3}}{\partial h}(h ; \phi)+\frac{\partial H}{\partial h}(h ; \phi)=0 .
$$


The functions $F_{i}(h ; \phi)(i=1,2,3)$ and $H(h ; \phi)$ are of course arbitrary, and it is understood that (7.16) defines $h\left(x_{1}, x_{2}, x_{3} ; \phi\right)$ which by substitution in (7.15) then determines $\phi\left(x_{1}, x_{2}, x_{3}\right)$ implicitly. As in the two dimensional case, the non degeneracy condition (3.6) excludes the solutions for $\phi$ which are independent of $x_{3}$. However, it is easy to show that the latter solutions as well as all those obtained from (7.11) and (7.12) through the C-map may all be constructed from (7.15) and (7.16). Note also how the equation (5.35), which defines implicit solutions to the Bateman equation in any dimension, is clearly a particular reduction of (7.15) and (7.16) in this case, corresponding to functions $F_{i}(h ; \phi)(i=1,2,3)$ and $H(h ; \phi)$ which are independent of $h$.

Moreover, the expressions (7.15) and (7.16) also lead to an interesting geometrical interpretation. Given a solution $\phi\left(x_{1}, x_{2}, x_{3}\right)$, consider the equation $\left(\phi\left(x_{1}, x_{2}, x_{3}\right)=\phi_{0}\right)$ with $\phi_{0}$ being an arbitrary constant. Such an equation defines a particular two dimensional surface in the space parametrised by $\left(x_{1}, x_{2}, x_{3}\right)$. From (7.15) and (7.16), one sees that this surface is the locus of the lines of intersection of the two planes defined by these two conditions as the parameter $h$ varies, or else, the surface is the plane defined by $(7.15)$ and (7.16) must then be either trivial (corresponding to $F_{i}(h ; \phi)(i=1,2,3)$ and $H(h ; \phi)$ independent of $h$ ) or proportional to (7.15) (corresponding to $F_{i}(h ; \phi)(i=1,2,3)$ and $H(h ; \phi)$ being all proportional to the same exponential function $(\exp (\alpha(\phi) h)))$. Thus, the surface defined by $\left(\phi\left(x_{1}, x_{2}, x_{3}\right)=\phi_{0}\right)$ is always a ruled surface known ${ }^{[11]}$ as a developable surface, namely a surface which may be developed onto the plane. These surfaces are either planes, cones, cylinders or tangent surfaces to curves (i.e. the locus of tangents to a curve). Indeed, the characteristic function $\left(F\left(x_{1}, x_{2}, x_{3}\right)=0\right)$ for a developable surface in three dimensions is precisely always a solution to the three dimensional Bateman equation $^{[11]}$. Actually, by considering the inverse C-map composed with the R-map, it may be seen that for any given functions $F_{i}(h ; \phi)(i=1,2,3)$ and $H(h ; \phi)$ in $(7.15)$ and (7.16), the surfaces defined by $\left(\phi\left(x_{1}, x_{2}, x_{3}\right)=\phi_{0}\right)$ and by (7.13) and (7.14) with $(F(h)=$ $\left.-F_{1}\left(h ; \phi_{0}\right) / F_{3}\left(h ; \phi_{0}\right)\right),\left(G(h)=-F_{2}\left(h ; \phi_{0}\right) / F_{3}\left(h ; \phi_{0}\right)\right)$ and $\left(H(h)=-H\left(h ; \phi_{0}\right) / F_{3}\left(h ; \phi_{0}\right)\right)$ are the same surfaces. Thus, all solutions to the universal string equations (1.3) in three spacetime dimensions are ${ }^{[2]}$ all developable surfaces. Note also that similar considerations apply in the case $(d=1)$, developable surfaces being then simply replaced by straight lines in a two dimensional space.

The results above have thus shown how it is possible in principle to find all solutions to $(1.1)-(1.3)$ for $(d=0,1,2)$, thereby establishing their implicit integrability. The situation for higher values of $d$ is left as an open question interesting in its own right. In this context, one may wonder what type of hypersurface would generalise the rôle played by developable surfaces above. Hereafter, general classes of solutions to (1.1) - (1.3) for arbitrary $d$ are presented, but we do not yet know how to construct, even implicitly, all solutions. However, the other instance where the numbers of universal equations of motion and fields are the same - accounting for Ward identities in the reparametrisation invariant case -, namely (6.6), (6.16) and (6.29), is very easily solved. The general solution to (6.6) is of course

$$
\phi^{a}(x)=A^{a} x+B^{a},
$$

with $A^{a}$ and $B^{a}$ being arbitrary constants. Using the C-map and accounting for the 
degenerate cases not obeying (3.6), the general solution to (6.16) is implicitly given by

$$
x_{i} F_{i}^{a}\left(\phi^{b}\right)+H^{a}\left(\phi^{b}\right)=0
$$

where $F_{i}^{a}\left(\phi^{b}\right)$ and $H^{a}\left(\phi^{b}\right)$ are arbitrary functions of the fields. Thus in this case, the constraints (1.6) define in fact all solutions to the generalised Bateman equation (1.5) with $(D=d-1)$. Finally, using the R-map and accounting for the condition (4.4), all solutions to (6.29) are obtained as

$$
\phi^{a}(x)=A^{a} f(x)+B^{a},
$$

where $f(x)$ is of course an arbitrary function.

Let us now present some classes of solutions to (1.1) - (1.3) for arbitrary $d$. Obviously, whenever the field $\phi$ or one of the fields $\phi^{a}$ in case 3) are independent of at least one of the coordinates, we always have a solution for either of the three equations. Consider now the generic universal equation (1.1). A large class of solutions is given by

$$
\phi\left(x_{i}\right)=G\left(x_{i} A_{i n}+B_{n}\right)+H
$$

where $A_{i n}, B_{n}$ and $H$ are arbitrary constants and $G\left(t_{n}\right)$ is an arbitrary function of $N$ arguments $t_{n}$ with $(N<d)$. In fact, the function (7.20) also defines a solution when $(N=d)$ provided either the then square matrix $A_{i n}$ or the matrix $\left(\partial^{2} G\left(t_{n}\right) / \partial t_{n} \partial t_{m}\right)$ have a vanishing determinant (for all values of $t_{n}$ in the latter case; any homogeneous function $G\left(t_{n}\right)$ of weight one is such an example). Whatever the value of $N,(7.20)$ is such that $\phi\left(x_{i}\right)$ is in fact constant along at least one of the directions in the space parametrised by the coordinates $x_{i}$. This situation is thus very similar to the one described above concerning developable surfaces.

Given a specific solution to (1.1) in $d$ dimensions, it is also possible to construct iteratively solutions to (1.1) in higher dimensions. Namely, if $\phi^{(d)}\left(x_{i}\right)$ is a solution in $d$ dimensions, the function defined by

$$
\phi^{(d+1)}\left(x_{i}, x_{d+1}\right)=\exp \left[\phi^{(d)}\left(x_{i}\right)+\alpha x_{d+1}+\beta\right]
$$

where $\alpha$ and $\beta$ are arbitrary constants, is always a solution to $(1.1)$ in $(d+1)$ dimensions. In particular, since all solutions to $(1.1)$ for $(d=2)$ are known, this procedure enables one to find large classes a solutions in higher dimensions. Finally, we should also mention that using the inverse C-map, it is possible to obtain still further solutions to (1.1) from the solutions to the Bateman equation given hereafter.

Let us now turn ${ }^{[1]}$ to the Bateman equation (1.2) in $d$ dimensions. As already mentioned, any field configuration obeying the relation (5.34), thus in particular those functions defined implicitly by (5.35), always provide solutions. Also, any function $\phi\left(x_{i}\right)$ homogeneous in the variables $x_{i}$ and of zero weight, is always a solution. Finally, the functions

$$
\phi\left(x_{i}\right)=\prod_{n=1}^{N}\left[x_{i} A_{i n}+B_{n}\right]^{\alpha_{n}}
$$


where $A_{i n}, B_{n}$ and $\alpha_{n}$ are arbitrary parameters, are always solutions provided $(N<d)$. If $(N=d)$, we must have either $\left(\operatorname{det} A_{i n}=0\right)$ or $\left(\sum_{n=1}^{N} \alpha_{n}=0\right)$.

Given a solution to (1.2) for a specific dimension, it is also possible ${ }^{[1]}$ to construct by iteration solutions in higher dimensions. Namely, if $\phi^{(d)}\left(x_{i}\right)$ is a solution in $d$ dimensions, solutions in $(d+1)$ dimensions are obtained from

$$
\phi^{(d+1)}\left(x_{i}, x_{d+1}\right)=\phi^{(d)}\left(x_{i}+h_{i}\left(x_{d+1}\right)\right),
$$

where $h_{i}\left(x_{d+1}\right)$ are arbitrary functions. However, this recursive procedure is different from the one obtained from (7.21) through the C-map (the description of the latter is left to the reader). Thus, using the C-map and its inverse, (7.21) and (7.23) provide two different iterative procedures for constructing new solutions in higher dimensions from specific ones in any particular dimension.

Finally, consider the C-map applied to the solutions in (7.20) for the universal equation of the generic hierarchy. This leads to the following implicit definition of solutions to the Bateman equation which are not independent of $x_{d}$

$$
x_{d}=G\left(x_{\alpha} A_{\alpha n}(\phi)+B_{n}(\phi)\right)+H(\phi) .
$$

Here, $A_{\alpha n}(\phi), B_{n}(\phi)$ and $H(\phi)$ are arbitrary functions of $\phi,(\alpha=1,2, \cdots, d-1)$ and $G\left(t_{n}\right)$ are arbitrary functions of $N$ arguments $t_{n}(n=1,2, \cdots, N)$ with $(N<(d-1))$. When $(N=d-1)$, we must have either $\left(\operatorname{det} A_{\alpha n}=0\right)$ or $\left(\operatorname{det} \partial^{2} G\left(t_{n}\right) / \partial t_{n} \partial t_{m}=0\right)$ as discussed after (7.20). Clearly, the definition (7.24) includes (5.35) as a special case, the latter corresponding to having $(N=1)$ and $G$ a linear function of its single argument. Incidentally, the latter case, which corresponds to applying the inverse C-map on (5.35), leads to the following obvious solutions to (1.1)

$$
\phi\left(x_{i}\right)=x_{i} A_{i}+B,
$$

with $A_{i}$ and $B$ arbitrary constants. Consequently, we have

$$
\phi_{i j}=0,
$$

which not only solves (1.1) most trivially, but also explains why (5.35) always defines field configurations $\phi\left(x_{i}\right)$ which in fact trivially solve all the equations of motion of the Bateman hierarchy, and not only the Bateman equation itself - a result pointed out after (5.35).

Let us conclude with some solutions to the generalised universal Bateman equations (1.5), and their dual representation (6.5) in the generalised generic hierarchy. Extending (5.34), field configurations obeying identities of the form

$$
\phi_{i j}^{a}=S_{i}^{a b} \phi_{j}^{b}+S_{j}^{a b} \phi_{i}^{b}
$$

for some coefficients $S_{i}^{a b}$, are always ${ }^{[1]}$ solutions to (1.5). In particular, this includes fields defined implicitly by the constraints

$$
x_{i} F_{i}^{a}\left(\phi^{b}\right)+H^{a}\left(\phi^{a}\right)=0
$$


with $F_{i}^{a}\left(\phi^{b}\right)$ and $H^{a}\left(\phi^{b}\right)$ being arbitrary functions. Such solutions are the generalisation of (5.35) already mentioned in the introduction. Actually, it is easy to verify that configurations obeying (7.27) also lead to

$$
Y_{\alpha \beta}^{a}=0
$$

with $Y_{\alpha \beta}^{a}$ defined in (3.9). This result shows that in fact (7.27) defines solutions to all equations of motion of the generalised Bateman hierarchy (6.10), and not only to the generalised Bateman equation (6.15), thus extending the similar result for the Bateman hierarchy for one field mentioned after (5.35).

It is also instructive to apply the inverse C-map to (7.28). One then obtains the following trivial solutions to $(6.5)$

$$
\phi^{a}\left(x_{i}\right)=x_{i} F_{i}^{a}+H^{a},
$$

with $F_{i}^{a}$ and $H^{a}$ now being arbitrary constants. Since we then have $\left(\phi_{i j}^{a}=0\right)$, it is obvious why (7.28) always defines solutions to all equations of motion for both the generalised generic and Bateman hierarchies (6.2) and (6.10). Clearly, the same applies to (7.27) since $Y_{\alpha \beta}^{a}$ then vanishes identically. In fact, in view of (7.20), (7.30) suggests an obvious generalisation. Namely, consider the fields

$$
\phi^{a}\left(x_{i}\right)=G^{a}\left(x_{i} A_{i n}+B_{n}\right)+H^{a},
$$

where $A_{i n}, B_{n}$ and $H^{a}$ are arbitrary coefficients and $G^{a}\left(t_{n}\right)$ are arbitrary functions of $N$ arguments $t_{n}$ with $(N<d)$. If $(N=d)$, we must also have $\left(\operatorname{det} A_{i n}=0\right)$. These field configurations, clearly generalising (7.30), are always solutions to the universal equations of motion (6.5). Using the C-map, one then also obtains the following constraints defining implicitly solutions to the generalised Bateman equations (6.15)

$$
y^{a}=G^{a}\left(x_{\alpha} A_{\alpha n}\left(\phi^{b}\right)+B_{n}\left(\phi^{b}\right)\right)+H^{a}\left(\phi^{b}\right) .
$$

Here, $A_{\alpha n}\left(\phi^{b}\right), B_{n}\left(\phi^{b}\right), H^{a}\left(\phi^{b}\right)$ and $G^{a}\left(t_{n}\right)$ are arbitrary functions with $(n=1,2, \cdots, N)$ and $(N<(d-D))$. If $(N=d-D)$, we must also have (det $\left.A_{\alpha n}\left(\phi^{b}\right)=0\right)$ (the coordinates $x_{i}$ have been split into $q$ coordinates $x_{\alpha}$ and $p$ coordinates $y^{a}$, where $p$ is the number of fields $\phi^{a}$ and $(d=p+q)$; see sect.3)). The solutions to the generalised Bateman equation (1.5) defined by the constraints (7.32) extend those provided by (7.28) but do not obey (7.27), so that such configurations are no longer solutions to all the other equations of motion of the generalised Bateman hierarchy. Finally, using the R-map, it should be clear how (7.31) also defines solutions to the reparametrisation invariant universal string and membrane equations of motion (6.27). As in (7.20), one then finds string and membrane configurations which are constant along at least one of the directions in spacetime, a situation which is not avoided even when implementing the iterative procedure (7.21). This class of solutions is a straightforward extension of developable surfaces to higher dimensions. However, it is not clear at all whether all solutions to the generalised universal equations of this paper would be of this type. 


\section{Conclusions}

The results of this paper have used two main ingredients. On the one hand, a triangular duality relationship between certain classes of arbitrary field theories, of classical topological field theories and of new types of string and membrane theories. On the other hand, the existence of a generic finite Euler hierarchy of Lagrangians and their equations of motion leading to a universal equation for one field in arbitrary dimensions which generalises the Plebanski equation ${ }^{[3]}$ for self-dual gravity in four dimensions.

The duality maps have made explicit the close similarity between reparametrisation invariant theories such as strings and membranes, and classical topological field theories whose space of classical solutions falls into diffeomorphic topological classes of the target manifold. The generic hierarchy has shown that there is a close connection - not yet properly understood - between the finiteness of a Euler hierarchy, the universality of its last non trivial equations of motion, and the fact that these equations of motion, even though non linear in the fields, are multilinear in their second derivatives and of order equal to the level at which they arise in the hierarchy.

The universal equations of motion have the peculiarity that they derive from an infinity of inequivalent Lagrangians and are covariant under arbitrary linear transformations both in base space and in target space, even though the associated Lagrangians do not necessarily possess these symmetries. Moreover, the infinity of Lagrangians sharing the same equations of motion strongly suggests the possible integrability of the universal equations of motion. This is indeed confirmed in the simplest cases where the complete solution to the equations can be specified using simple methods.

These results obviously raise a series of interesting questions. Clearly, one may wonder whether there exist finite Euler hierarchies leading to universal equations of motion other than the generic hierarchy and all its descendants constructed here. Could such hierarchies also exist for anticommuting fields? Is the supersymmetrisation of the present hierarchies possible while preserving their finiteness and universality? Do such finite Euler hierarchies exhaust all classes of field equations admitting an infinity of inequivalent Lagrangians? In any case, were such new hierarchies to exist, one would also obtain their dual representations as new classical topological field theories and new string and membrane theories.

The question of integrability of the universal equations of this paper - and of any others were they to exist - also begs for an answer, which may well turn out to have profound consequences for our understanding of integrable systems in higher dimensions.

Of course, there is also the whole problem of quantising the infinity of field theories leading to these universal equations of motion. In the context of their "Bateman representation" as classical topological field theories, it would certainly be of interest to find out what becomes of the classical solutions once these systems are quantised, since such systems possess a novel kind of gauge symmetry only realised on the space of classical solutions but not at the level of the action. In the context of the string or membrane representation, the whole issue of a consistent BRST quantisation ${ }^{[10]}$ of these gauge invariant theories certainly warrants a close examination, having in mind among other issues the 
possibility of critical dimensions and the question of physical states.

On the other hand, the generic hierarchy uses the Euler operator $\mathcal{E}$ as an essential ingredient in its construction. In fact, this operator is nilpotent, i.e. $\left(\mathcal{E}^{2}=0\right)$, when acting on Lagrangians having a dependence only on derivatives of fields but not on the fields themselves, as is the case here. In other words, this operator is in our case ${ }^{[1]}$ in the nature of the exterior derivative in differential geometry or the BRST charge in gauge invariant systems. It is not clear whether the associated cohomological structure inherent ${ }^{[1]}$ to the construction of Euler hierarchies plays any crucial rôle in the existence of the generic finite hierarchy. Nevertheless, it certainly raises the question whether other finite Euler hierarchies could not be constructed on the basis of a modified Euler operator more in the nature of a covariant exterior derivative for some principal fiber bundle or some generalisation thereof in the context of infinite dimensional manifolds of field configurations.

Finally, there is also the issue of the possible physical relevance of these universal equations. The obvious suggestion is in the context of theories for gravity. On the one hand, the fields of a classical topological field theory may be thought of as spacetime coordinates, since classical solutions are covariant under arbitrary field redefinitions. In this respect, the quantisation of these theories becomes more pressing. On the other hand, in view of the situation with ordinary critical strings based on the Nambu-Goto action and its different supersymmetric extensions, reparametrisation invariant universal equations could possibly also be of relevance to theories of gravity. Here again, the understanding of the space of physical states at the quantum level is required. However, one may also wonder whether the universal equations of this paper have any other connection with present developments of string theory, either in the context of the geometrical realisation of $\mathrm{W}$-algebras ${ }^{[12]}$ or indeed of $\mathrm{W}$-gravity theories ${ }^{[13]}$, or in the context of the matrix model formulation $^{[14,15,16]}$ of two dimensional quantum gravity and non critical string theories, this latter formulation being directly related to two dimensional integrable systems such as the $\mathrm{KdV}$ hierarchy which indeed made its appearance here as well.

\section{Acknowledgement}

The work of J.G. is supported through a Senior Research Assistant position funded by the S.E.R.C. 


\section{Appendix}

In complement to Sect.5, giving the proof for the generic finite Euler hierarchy for one field, definitions and some properties of generalised determinant and trace functions of matrices are collected in this Appendix. These generalised determinants are multilinear functions of collections of arbitrary $d \times d$ matrices $A_{i j}^{(p)}(p=1,2, \ldots ; i, j=1,2, \cdots, d)$. These functions include as particular cases the ordinary determinant and trace functions when all matrices $A_{i j}^{(p)}$ are identical.

First, for any $(0 \leq n \leq d)$, let us introduce the following quantities

$$
\begin{aligned}
T_{i_{1} \cdots i_{n} ; j_{1} \cdots j_{n}}^{(n)} & =\sum_{\sigma_{n}}\left|\sigma_{n}\right| \delta_{i_{1} \sigma_{n}\left(j_{1}\right)} \cdots \delta_{i_{n} \sigma_{n}\left(j_{n}\right)} \\
& =\frac{1}{(d-n) !} \epsilon_{i_{1} \cdots i_{d}} \epsilon_{j_{1} \cdots j_{d}} \delta_{i_{n+1} j_{n+1}} \cdots \delta_{i_{d} j_{d}} .
\end{aligned}
$$

In this definition, the summation is over all permutations $\sigma_{n}$ of $n$ elements, and $\left|\sigma_{n}\right|$ denotes the signature of each permutation. In particular, we have $T^{(0)}=1$. Note that the quantities $T_{i_{1} \cdots i_{n} ; j_{1} \cdots j_{n}}^{(n)}$ are symmetric in the n-plets $\left(i_{1} \cdots i_{n}\right)$ and $\left(j_{1} \cdots j_{n}\right)$ and antisymmetric in the indices $i_{k}$ and $j_{k}$ separately. They also obey the identities

$$
\delta_{i_{n+1} j_{n+1}} T_{i_{1} \cdots i_{n+1} ; j_{1} \cdots j_{n+1}}^{(n+1)}=(d-n) T_{i_{1} \cdots i_{n} ; j_{1} \cdots j_{n}}^{(n)}
$$

and

$$
T_{i_{1} \cdots i_{n+1} ; j_{1} \cdots j_{n+1}}^{(n+1)}=T_{i_{1} \cdots i_{n} ; j_{1} \cdots j_{n}}^{(n)} \delta_{i_{n+1} j_{n+1}}-\sum_{p=1}^{n} T_{i_{1} \cdots i_{n} ; j_{1} \cdots j_{p-1} j_{n+1} j_{p+1} \cdots j_{n}}^{(n)} \delta_{i_{n+1} j_{p}} .
$$

The second of these relations, which is the converse of the first, is the combinatorial property at the origin of the generic hierarchy of Sect.5.

Given a collection of arbitrary matrices $A_{i j}^{(p)}(p=1,2, \cdots, n)$ with $(0 \leq n \leq d)$, consider now the multilinear functions defined by

$$
L_{i_{n+1} \cdots i_{d} ; j_{n+1} \cdots j_{d}}^{(n)}\left(A^{(1)}, \cdots, A^{(n)}\right)=\frac{1}{n !(d-n) !} \epsilon_{i_{1} \cdots i_{d}} \epsilon_{j_{1} \cdots j_{d}} A_{i_{1} j_{1}}^{(1)} \cdots A_{i_{n} j_{n}}^{(n)}
$$

and

$$
\begin{aligned}
\operatorname{Tr}^{(n)}\left(A^{(1)}, \cdots, A^{(n)}\right) & =L_{i_{n+1} \cdots i_{d} ; i_{n+1} \cdots i_{d}}^{(n)}\left(A^{(1)}, \cdots, A^{(n)}\right) \\
& =\frac{1}{n !} T_{i_{1} \cdots i_{n} ; j_{1} \cdots j_{n}}^{(n)} A_{i_{1} j_{1}}^{(1)} \cdots A_{i_{n} j_{n}}^{(n)}
\end{aligned}
$$

When all arguments $A^{(p)}$ are identical, these functions will be denoted $L_{i_{n+1}}^{(n)}$ and $\operatorname{Tr}^{(n)}(A)$ respectively. Note that we have

$$
\operatorname{Tr}^{(1)}(A)=\operatorname{tr} A, \quad \operatorname{Tr}^{(d)}(A)=\operatorname{det} A=L^{(d)}(A),
$$


showing that the functions (A.4) and (A.5) indeed generalise the ordinary trace and determinant functions of matrices. In fact, the functions $L_{i_{n+1} \cdots i_{d} ; j_{n+1} \cdots j_{d}}^{(n)}(A)$ essentially correspond to the minors of order $n$ of the matrix $A$.

Clearly, the functions $L^{(n)}$ and $\operatorname{Tr}^{(n)}$ are fully symmetric functions of their arguments $A^{(p)}(p=1,2, \cdots, n)$. Also, the functions $L_{i_{n+1} \cdots i_{d} ; j_{n+1} \cdots j_{d}}^{(n)}\left(A^{(1)}, \cdots, A^{(n)}\right)$ are antisymmetric under the exchange of any pair of the $i_{k}$ or of the $j_{k}$ indices. Moreover, these functions are symmetric under the exchange of the $(d-n)$-plets $\left(i_{n+1} \cdots i_{d}\right)$ and $\left(j_{n+1} \cdots j_{d}\right)$ only if all the arguments $A^{(p)}(p=1,2, \cdots, n)$ are symmetric matrices. Finally, we have the following relations and particular cases

$$
\begin{gathered}
L_{i_{n+1} \cdots i_{d} ; j_{n+1} \cdots j_{d}}^{(n)}\left(A^{(1)^{\mathrm{T}}}, \cdots, A^{(n)^{\mathrm{T}}}\right)=L_{j_{n+1} \cdots j_{d} ; i_{n+1} \cdots i_{d}}^{(n)}\left(A^{(1)}, \cdots, A^{(n)}\right), \\
\operatorname{Tr}^{(n)}\left(A^{(1)^{\mathrm{T}}}, \cdots, A^{(n)^{\mathrm{T}}}\right)=\operatorname{Tr}^{(n)}\left(A^{(1)}, \cdots, A^{(n)}\right), \\
L_{i_{1} \cdots i_{d} ; j_{1} \cdots j_{d}}^{(0)}=\frac{1}{d !} \epsilon_{i_{1} \cdots i_{d}} \epsilon_{j_{1} \cdots j_{d}}=\frac{1}{d !} T_{i_{1} \cdots i_{d} ; j_{1} \cdots j_{d}}^{(d)}, \\
L^{(d)}\left(A^{(1)}, \cdots, A^{(d)}\right)=\operatorname{Tr}^{(d)}\left(A^{(1)}, \cdots, A^{(d)}\right) .
\end{gathered}
$$

Generalising the expansion of the ordinary determinant function in terms of minors of a matrix, we also have the following identities for any $p<n$

$$
\begin{aligned}
& L_{i_{n+1} \cdots i_{d} ; j_{n+1} \cdots j_{d}}^{(n)}\left(A^{(1)}, \cdots, A^{(n)}\right)= \\
& \quad=\frac{p !(d-p) !}{n !(d-n) !} L_{i_{p+1} \cdots i_{d} ; j_{p+1} \cdots j_{d}}^{(p)}\left(A^{(1)}, \cdots, A^{(p)}\right) A_{i_{p+1} j_{p+1}}^{(p+1)} \cdots A_{i_{n} j_{n}}^{(n)},
\end{aligned}
$$

and

$$
\begin{aligned}
& \operatorname{Tr}^{(n)}\left(A^{(1)}, \cdots, A^{(n)}\right)= \\
& \quad=\frac{p !(d-p) !}{n !(d-n) !} L_{i_{p+1} \cdots i_{d} ; j_{p+1} \cdots j_{d}}^{(p)}\left(A^{(1)}, \cdots, A^{(p)}\right) A_{i_{p+1} j_{p+1}}^{(p+1)} \cdots A_{i_{n} j_{n}}^{(n)} \delta_{i_{n+1} j_{n+1}} \cdots \delta_{i_{d} j_{d}} .
\end{aligned}
$$

In particular, since $\operatorname{Tr}^{(d)}(A)=\operatorname{det} A=L^{(d)}(A)$, the last identity shows that

$$
L_{i ; j}^{(d-1)}(A)=\widetilde{A}_{j i}
$$

where $\widetilde{A}_{i j}$ is the adjugate matrix of $A_{i j}$ given by

$$
\widetilde{A}=(\operatorname{det} A) A^{-1} .
$$

Finally, generalising the well known result for ordinary determinants, we have the multiplication theorems

$$
\begin{aligned}
& L_{i_{n+1} \cdots i_{d} ; j_{n+1} \cdots j_{d}}^{(n)}\left(A^{(1)} B, \cdots, A^{(n)} B\right)= \\
& \quad=L_{i_{n+1} \cdots i_{d} ; k_{n+1} \cdots k_{d}}^{(n)}\left(A^{(1)}, \cdots, A^{(n)}\right) L_{k_{n+1} \cdots k_{d} ; j_{n+1} \cdots j_{d}}^{(n)}(B) \\
& L_{i_{n+1} \cdots i_{d} ; j_{n+1} \cdots j_{d}}^{(n)}\left(B A^{(1)}, \cdots, B A^{(n)}\right)= \\
& \quad=L_{i_{n+1} \cdots i_{d} ; k_{n+1} \cdots k_{d}}^{(n)}(B) L_{k_{n+1} \cdots k_{d} ; j_{n+1} \cdots j_{d}}^{(n)}\left(A^{(1)}, \cdots, A^{(n)}\right),
\end{aligned}
$$


and

$$
\begin{aligned}
& \operatorname{Tr}^{(n)}\left(A^{(1)} B, \cdots, A^{(n)} B\right)=\operatorname{Tr}^{(n)}\left(B A^{(1)}, \cdots, B A^{(n)}\right)= \\
& \quad=L_{i_{n+1} \cdots i_{d} ; j_{n+1} \cdots j_{d}}^{(n)}\left(A^{(1)}, \cdots, A^{(n)}\right) L_{j_{n+1} \cdots j_{d} ; i_{n+1} \cdots i_{d}}^{(n)}(B) .
\end{aligned}
$$

In particular, we thus obtain

$$
\begin{aligned}
\operatorname{Tr}^{(d)}\left(A^{(1)} B, \cdots, A^{(d)} B\right) & =\operatorname{Tr}^{(d)}\left(B A^{(1)}, \cdots, B A^{(d)}\right)= \\
& =(\operatorname{det} B) \operatorname{Tr}^{(d)}\left(A^{(1)}, \cdots, A^{(d)}\right),
\end{aligned}
$$

and

$$
\begin{aligned}
\operatorname{Tr}^{(d-1)}\left(A^{(1)} B, \cdots, A^{(d-1)} B\right) & =\operatorname{Tr}^{(d-1)}\left(B A^{(1)}, \cdots, B A^{(d-1)}\right)= \\
& =\widetilde{B}_{i j} L_{i ; j}^{(d-1)}\left(A^{(1)}, \cdots, A^{(d-1)}\right),
\end{aligned}
$$

where $\widetilde{B}$ is the adjugate of $B$ (see (A.14)). Note that these results, which are rather easy to prove, require that the same matrice $B$ multiplies the matrices $A^{(p)}$.

As we have seen, the functions $\operatorname{Tr}^{(d)}=L^{(d)}$ generalise the ordinary determinant of a $d \times d$ matrix to the case of $d$ such matrices. As is well known, the determinant of a matrix vanishes whenever at least one of its lines (or columns) is a linear combination of the other lines (or columns). More generally here, we have that

$$
\operatorname{Tr}^{(d)}\left(A^{(1)}, \cdots, A^{(d)}\right)=L^{(d)}\left(A^{(1)}, \cdots, A^{(d)}\right)=0
$$

whenever all matrices $A^{(p)}(p=1,2, \cdots, d)$ have a vanishing determinant and for each one of them, the same linear combination of its lines (or columns), with the same coefficients for all matrices, applies. The converse is also true, at least when all matrices which are different among the set of $d$ matrices are also linearly independent.

The proof of this statement is straightforward. For definiteness, let us assume that it is the last column of each matrix which is a linear combination of its other columns, namely

$$
A_{i d}^{(p)}=\sum_{\beta=1}^{d-1} A_{i \beta}^{(p)} C_{\beta}, \quad \text { for } \quad p=1,2, \cdots, d .
$$

The coefficients $C_{\beta}$ are thus the same for all matrices $A^{(p)}(\alpha, \beta=1,2, \cdots,(d-1))$. Given (A.20), we actually have

$$
A_{i j}^{(p)}=\left(\begin{array}{cc}
A_{\alpha \beta}^{(p)} & A_{\alpha \beta}^{(p)} C_{\beta} \\
A_{d \beta}^{(p)} & A_{d \beta}^{(p)} C_{\beta}
\end{array}\right)=\left(\begin{array}{cc}
A_{\alpha \beta}^{(p)} & 0 \\
A_{d \beta}^{(p)} & 0
\end{array}\right)\left(\begin{array}{cc}
\delta_{\alpha \beta} & C_{\alpha} \\
0 & 0
\end{array}\right) .
$$

Here, $i$ or $\alpha$ and $j$ or $\beta$ denote line and column indices respectively. Using the multiplication theorem (A.17), it is clear that (A.19) is indeed obtained.

To conclude, let us now consider matrices of the form

$$
A_{i j}^{(p)}=\left(\begin{array}{cc}
A_{\alpha \beta}^{(p)} & A_{\alpha \beta}^{(p)} C_{\beta} \\
C_{\alpha} A_{\alpha \beta}^{(p)} & C_{\alpha} A_{\alpha \beta}^{(p)} C_{\beta}
\end{array}\right)
$$


where $C_{\alpha}$ are arbitrary coefficients common to all matrices $A_{i j}^{(p)}(p=1,2, \cdots, d)$ (the notation is the same as in (A.20)). From the previous result, we know that the functions $\operatorname{Tr}^{(d)}=L^{(d)}$ then vanish for these matrices. However, the functions $\operatorname{Tr}^{(d-1)}$ and $L_{i ; j}^{(d-1)}$ need not vanish - in the same way that the minors of order $(d-1)$ of a $d \times d$ matrix of vanishing determinant need not vanish. Given (A.22), we have in fact

$$
A_{i j}^{(p)}=\left(\begin{array}{cc}
\delta_{\alpha \beta} & 0 \\
C_{\beta} & 0
\end{array}\right)\left(\begin{array}{cc}
A_{\alpha \beta}^{(p)} & 0 \\
0 & 0
\end{array}\right)\left(\begin{array}{cc}
\delta_{\alpha \beta} & C_{\alpha} \\
0 & 0
\end{array}\right)
$$

From a straightforward calculation, one now obtains

$$
\begin{aligned}
& L_{i ; j}^{(d-1)}\left(\left(\begin{array}{cc}
\delta_{\alpha \beta} & 0 \\
C_{\beta} & 0
\end{array}\right)\right)=\left(\begin{array}{cc}
0 & -C_{\alpha} \\
0 & 1
\end{array}\right), \\
& L_{i ; j}^{(d-1)}\left(\left(\begin{array}{cc}
\delta_{\alpha \beta} & C_{\alpha} \\
0 & 0
\end{array}\right)\right)=\left(\begin{array}{cc}
0 & 0 \\
-C_{\beta} & 1
\end{array}\right), \\
& L_{i ; j}^{(d-1)}\left(\left(\begin{array}{cc}
A_{\alpha \beta}^{(1)} & 0 \\
0 & 0
\end{array}\right), \cdots,\left(\begin{array}{cc}
A_{\alpha \beta}^{(d-1)} & 0 \\
0 & 0
\end{array}\right)\right)=\operatorname{Tr}_{(d-1)}^{(d-1)}\left(A_{\alpha \beta}^{(1)}, \cdots, A_{\alpha \beta}^{(d-1)}\right)\left(\begin{array}{cc}
0 & 0 \\
0 & 1
\end{array}\right) .
\end{aligned}
$$

In the last equality, the function $\operatorname{Tr}_{(d-1)}^{(d-1)}$ is a generalised determinant for $(d-1) \times(d-1)$ matrices. From these results, (A.23) and the multiplication theorem (A.15), one finally concludes that for matrices of the form (A.22), the functions $L_{i ; j}^{(d-1)}$ take the following values

$$
L_{i ; j}^{(d-1)}\left(A^{(1)}, \cdots, A^{(d-1)}\right)=\operatorname{Tr}_{(d-1)}^{(d-1)}\left(A_{\alpha \beta}^{(1)}, \cdots, A_{\alpha \beta}^{(d-1)}\right)\left(\begin{array}{cc}
C_{\alpha} C_{\beta} & -C_{\alpha} \\
-C_{\beta} & 1
\end{array}\right)
$$




\section{REFERENCES}

[1] D. B. Fairlie, J. Govaerts and A. Morozov, Universal Field Equations with Covariant Solutions, Durham preprint DTP-91/55, hepth-9110022 (October 1991), published in Nuclear Physics B.

[2] D. B. Fairlie and J. Govaerts, Universal Field Equations with Reparametrisation Invariance, Durham preprint DTP-92/11, hepth-9202056 (February 1992), to appear in Physics Letters B.

[3] J. D. Finley III and J. F. Plebanski, Jour. Math. Phys. 17 (1976) 585.

[4] H. Bateman, Proc. Roy. Soc. London A 125 (1929) 598.

[5] P. R. Garabedian, Partial Differential Equations (John Wiley \& Sons, New York, 1964) p. 517.

[6] E. Witten, Comm. Math. Phys. 117 (1988) 353; ibid. 118 (1988) 411.

[7] Y. Nambu, Lectures at the Copenhagen Symposium (1970).

[8] T. Goto, Prog. Theor. Physics 46 (1971) 1560.

[9] E. Olver, Applications of Lie Groups to Differential Equations, Graduate Texts in Mathematics 107 (Springer Verlag, Berlin, 1986) p. 252.

[10] For a recent review, see J. Govaerts, Hamiltonian Quantisation and Constrained Dynamics, Lecture Notes in Mathematical and Theoretical Physics 4 (Leuven University Press, Leuven, 1991).

[11] L. P. Eisenhart, An Introduction to Differential Geometry, (Princeton University Press, Princeton, 1940) p. 59.

[12] J.-L. Gervais and Y. Matsuo, Phys. Lett. B274 (1992) 309; preprint LPTENS-91/35, NBI-HE-91/50, hepth-9201026 (January 1992).

[13] C. M. Hull, Phys. Lett. B269 (1991) 257.

[14] E. Brezin and V. A. Kazakov, Phys. Lett. B236 (1990) 144.

[15] M. Douglas and S. Shenker, Nucl. Phys. B335 (1990) 635.

[16] D. Gross and A. A. Migdal, Phys. Rev. Lett. 64 (1990) 127; Nucl. Phys. B340 (1990) 333. 